\title{
Composable Behavioral Models and Schematic-Based Simulation of Electrokinetic Lab-on-a-Chip Systems
}

\author{
Yi Wang, Qiao Lin, and Tamal Mukherjee, Member, IEEE
}

\begin{abstract}
This paper presents composable behavioral models and a schematic-based simulation methodology to enable topdown design of electrokinetic (EK) lab-on-a-chip (LoC). Complex EK LoCs are shown to be decomposable into a system of elements with simple geometry and specific function. Parameterized and analytical models are developed to describe the electric and biofluidic behavior within each element. Electric and biofluidic pins at element terminals support the communication between adjacent elements in a simulation schematic. An analog hardware description language implementation of the models is used to simulate LoC subsystems for micromixing and electrophoretic separation. Both direct current (dc) and transient analysis can be performed to capture the influence of system topology, element sizes, material properties, and operational parameters on LoC system performance. Accuracy (relative error generally less than $5 \%)$ and speedup $(>100 \times)$ of the schematic-based simulation methodology are demonstrated by comparison to experimental measurements and continuum numerical simulation.
\end{abstract}

Index Terms-Behavioral model, dispersion, lab-on-a-chip, micromixing, schematic-based simulation, top-down design.

\section{NOMENCLATURE}

\section{Variables}

A Maximum concentration of species band.

$c \quad$ Concentration of species band.

$c_{m} \quad$ Channel width-averaged concentration of species band. ment of the species band.

$C_{e} \quad$ Electric conductivity of buffer, $\mathrm{S} / \mathrm{m}$.

$d_{n} \quad$ Mixing concentration coefficients.

$d_{m} \quad$ Mixing concentration coefficients at inlet of converging/diverging mixing intersection.

$D \quad$ Molecular diffusivity of species, $\mathrm{m}^{2} / \mathrm{s}$.

E Electric field strength, V/cm.

$h \quad$ Depth of microchannels, $\mu \mathrm{m}$.

I Electric current through buffer, A.

$L \quad$ Length of microchannels, $\mu \mathrm{m}$.

$L_{\text {det }} \quad$ Detector path length, $\mu \mathrm{m}$. $c_{p} \quad p$ th moment of the concentration in a longitudinal fila-

$m_{p} \quad p$ th moment of the widthwise average concentration of species band.

$N \quad$ Internal node of converging/diverging intersections.

$N_{\text {s }} \quad$ Plate number of separation.

Pe Peclet number.

$Q \quad$ Index of mixing residual of normalized sample concentration profile.

$q \quad$ Volumetric buffer flow rate, $\mathrm{m}^{3} / \mathrm{s}$.

$r_{c} \quad$ Mean radius of turn separation channels, $\mu \mathrm{m}$.

$R \quad$ Resistance of microfluidic elements, $\Omega$.

$s \quad$ Flow ratio, combing or splitting position.

$S_{n m} \quad$ Skew coefficients of species band.

$t \quad$ Separation time, s.

$\Delta t \quad$ Residence time of the centroid of species bands within channels, s.

Electrokinetic (EK) velocity of species band, $\mathrm{m} / \mathrm{s}$.

$U$ Widthwise average EK velocity of species band, $\mathrm{m} / \mathrm{s}$.

$V \quad$ Voltage at element terminals or reservoirs, V.

$w \quad$ Width of microchannels, $\mu \mathrm{m}$.

$y \quad$ Widthwise coordinate.

$z \quad$ Axial coordinate.

$\gamma \quad$ Factor of geometric effect on mixing.

$\chi \quad$ Normalized EK velocity of species band relative to the average.

$\eta \quad$ Normalized widthwise coordinate.

$\mu \quad$ EK mobility of sample/species, $\mathrm{m}^{2} /(\mathrm{V} \cdot \mathrm{s})$.

$\theta \quad$ Included angle of turn channels, $\mathrm{rad}$.

$\sigma^{2} \quad$ Variance of species band, $\mathrm{m}^{2}$.

$\tau \quad$ Dimensionless separation time.

$\xi \quad$ Normalized longitudinal coordinate.

Superscript/Subscript

$l \quad$ Quantities at the left inlet/outlet.

$r \quad$ Quantities at the right inlet/outlet.

in Quantities at the inlet.

out Quantities at the outlet.

det Quantities at the detector.

\section{INTRODUCTION}

Manuscript received February 15, 2005; revised June 10, 2005. This work was supported by the Defense Advanced Research Projects Agency (DARPA) and the Air Force Research Laboratory, Air Force Material Command, USAF, under Grant F30602-01-2-0587, and by the National Science Foundation (NSF) Information Technology Research (ITR) program under Award CCR-0325344. This paper was recommended by Associate Editor K. Chakrabarty.

Y. Wang and Q. Lin are with the Department of Mechanical Engineering, Carnegie Mellon University, Pittsburgh, PA 15213 USA.

T. Mukherjee is with the Department of Electrical and Computer Engineering, Carnegie Mellon University, Pittsburgh, PA 15213 USA.

Digital Object Identifier 10.1109/TCAD.2005.855942 
analysis speedup, extremely low biosample consumption, parallel processing capability, high levels of integration, and ease of automation. Integrated biofluidic LoCs that are based on EK transport of charged biomolecules and biofluids are of particular interest, as they are amenable to integration with EK injection, electrophoresis-based analysis, direct and accurate flow control [3], and electronics.

However, efficient modeling and simulation to assist designs of such biofluidic LoCs at the system level continue to be a challenge. This is due to the lack of an efficient design methodology to tackle the growing system complexity arising from two sources, namely: 1) more and more components are being integrated [4] and 2) components with diverse functionalities are being integrated [5]. An additional source of the complexity is the nature of the microscale multiphysics phenomena within LoCs (e.g., the turn-geometry-induced skew and broadening of the species band [6] and the slow molecular diffusion-based mixing [7]), which requires accurate models and simulation for iterative design studies. Presently, detailed numerical simulation [8], [9] is the only available way to obtain desired modeling accuracy. However, their central processing unit (CPU) time and memory requirements are prohibitive for system-level designs of complex LoCs. For example, a finiteelement-based simulation of a simple microchip consisting of a pair of complementary turns for electrophoretic separation applications can cost several hours to days [10]. Reduced-order macromodels have to be built from each numerical simulation and stitched together for an overall system simulation [11], [12]. The resulting macromodels in this bottom-up approach to design are specific to the geometry that was simulated numerically. Thus, the macromodels have to be regenerated whenever the geometry is perturbed for design optimization. This leads to unacceptably long design iterations and hinders the industrial application of this approach to LoC design. To address these issues, efficient parameterized modeling as well as system (circuit) level simulations has recently attracted a lot of attention. Qiao and Aluru [13] proposed a compact model to evaluate the flow rate and pressure distribution of both EK and pressuredriven flow within the network and capture the effect of the nonuniform zeta potential at the channel wall. Xuan and Li [14] later presented a fully analytical model to capture the effects of channel size and surface EK properties on microfluidic characteristics using phenomenological coefficients. Both these papers focus on bulk fluid flow in microchannels and ignore the details of sample transport that often become the limiting issues in biochip design. Coventor's circuit level microelectromechanical systems (MEMS) and microfluidics modeling and simulation environment, ARCHITECT [15], includes an EK library with simple models for injectors, straight channels and turns that can model sample transport in electrophoretic separation. However, it still requires users to extract parameters from full numerical simulations, which, hence, does not allow designs of electrophoresis channels of general shape where the interaction of dispersion effects between turns can be very strong and, hence, significantly limits its usefulness [10]. Zhang et al. [16] developed an integrated modeling and simulation environment for microfluidic systems in SystemC, which was used to evaluate and compare the performance of continuous-flow and droplet-based microfluidic systems on a polymerase chain reaction (PCR). Like the Coventor solution, the focus is at the system level, with an assumption that reduced-order models from detailed numerical simulation or experimental data are available. Most recently, Chatterjee and Aluru [17] combined circuit/device model to analyze fluidic transport, chemical reaction, reagent mixing as well as separation in integrated microfluidic systems. These models exploit an analogy between fluid and sample transport, effectively reducing the partial differential equations governing the problem into a single ordinary differential equation or an algebraic equation, leading to fast simulation times. However, this speedup is at the cost of ignoring local geometry-induced nonidealities.

This paper presents a top-down methodology that is both accurate and efficient in handling biofluidic LoC complex system designs. Based on the system hierarchy, we geometrically decompose a complex LoC into a collection of commonly used microfluidic channel elements. The design topology is captured by interconnecting these elements. Electrical and biofluidic information is exchanged between adjacent interconnected elements. Parameterized behavioral models are analytically derived to efficiently and accurately capture the multiphysics behavior of the elements. As a result, a complex LoC can be represented by a system-level schematic model that can be iteratively simulated to investigate the impact of a change in design topology, element sizes, and material properties on the overall LoC performance. Examples will focus on EK passive micromixers and electrophoretic separation chips that can work as independent biofluidic devices or serve as subsystems of an integrated LoC system.

Unlike the bottom-up design methodology, where reducedorder models are obtained from numerical simulations, use of parameterized models enables a top-down design methodology similar to system-on-a-chip (SOC) [18] and MEMS designs [19]. The top-down methodology (shown in Fig. 1) begins with a conceptual schematic representation of the system, gradually and hierarchically specifying components and elements of LoC using the behavioral models stored in the library. Then the schematic representation is used for iterative performance evaluation and design optimization. The design process ends with numerical simulations to verify that the design goals have been reached, and the design is finally sent to layout and fabrication.

In this paper, we will consider hierarchical schematic representation of LoCs (in particular, the microchips used for electrophoretic separation and micromixing) in Section II, including the system composition, operation, and hierarchy of the LoCs and the definition of pins and wires that enable the communication between the elements. A description of the behavioral models of the elements appears in Section III. The behavioral models are stored in model libraries and used for schematic simulations with examples shown in Section IV. Optimization-based synthesis involving iterative simulations is presented elsewhere [20], [21].

\section{SChematic RePRESENTATION}

In this section, we will first introduce the system composition and operation of a canonical $\mathrm{LoC}$, as well as the functionalities 


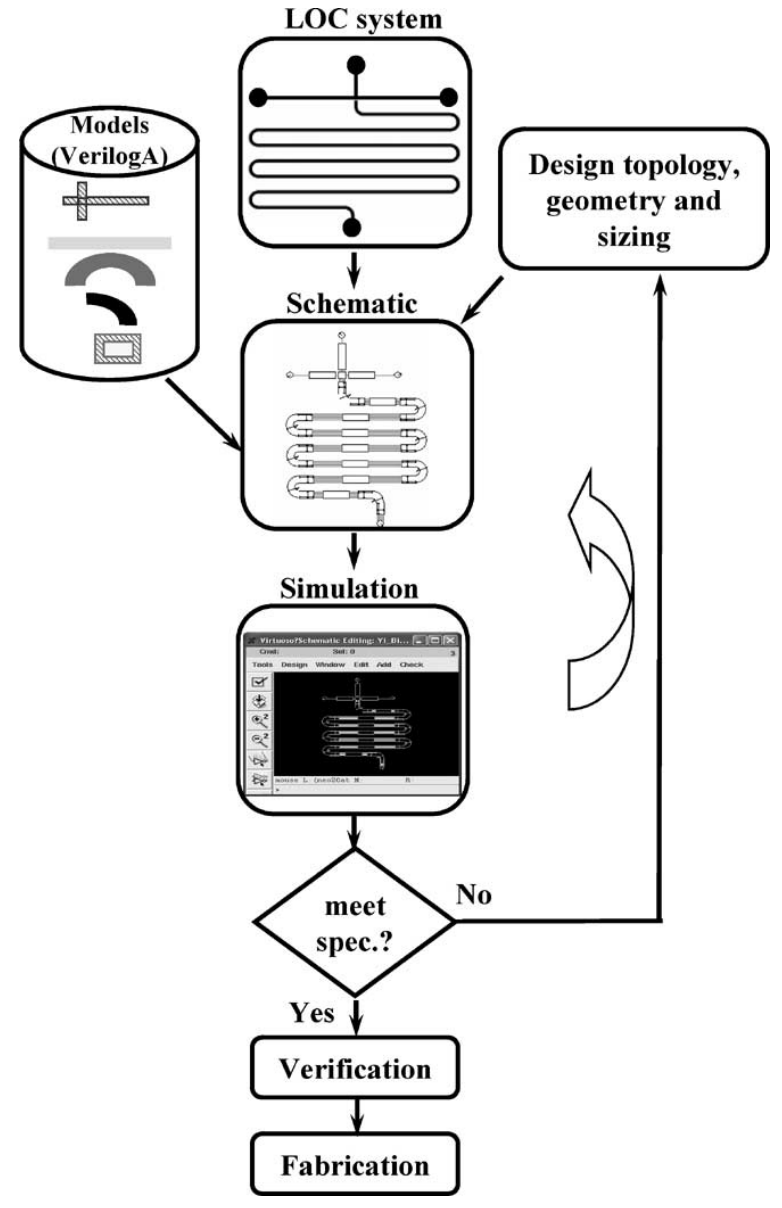

Fig. 1. Flowchart of the modeling and simulations of biofluidic LoC based on the top-down design methodology. A conceptual schematic representation of the system is generated by wiring element behavioral models in the library. Then the schematic representation is used for iterative performance evaluation and design optimization. The design process ends with numerical verification on the design goals, and the design is finally sent to layout and fabrication.

that can be achieved. Then we will illustrate the process of decomposing a complex LoC into commonly used biofluidic elements based on the geometrical and functional hierarchy of the LoC. Then electric and biofluidic pins, and analog wiring buses will be defined to link these elements and obtain a complete simulation schematic.

\section{A. LoC Introduction}

A variety of LoCs with diverse chemical and biological applications have been demonstrated to date. A canonical LoC integrating the functions of micromixing, reaction, injection, and separation is shown in Fig. 2. Its operation involves typical functions from a biochemical laboratory: synthesis and analysis. In the first phase, electrical voltages are applied between reservoirs 1-5. The electric field arising from these voltages moves the sample by EK flow [22], leading to dilution by the buffer solvent or mixing with the reagent or enzyme within the micromixer. The mixture then flows into a biochemical reactor. Reaction products are generated, often with the aid of external activation such as heat, light, or catalyst. Usually, the sample and reagent are continuously supplied by the reservoirs; therefore, the concentrations of all the samples, reagents, and

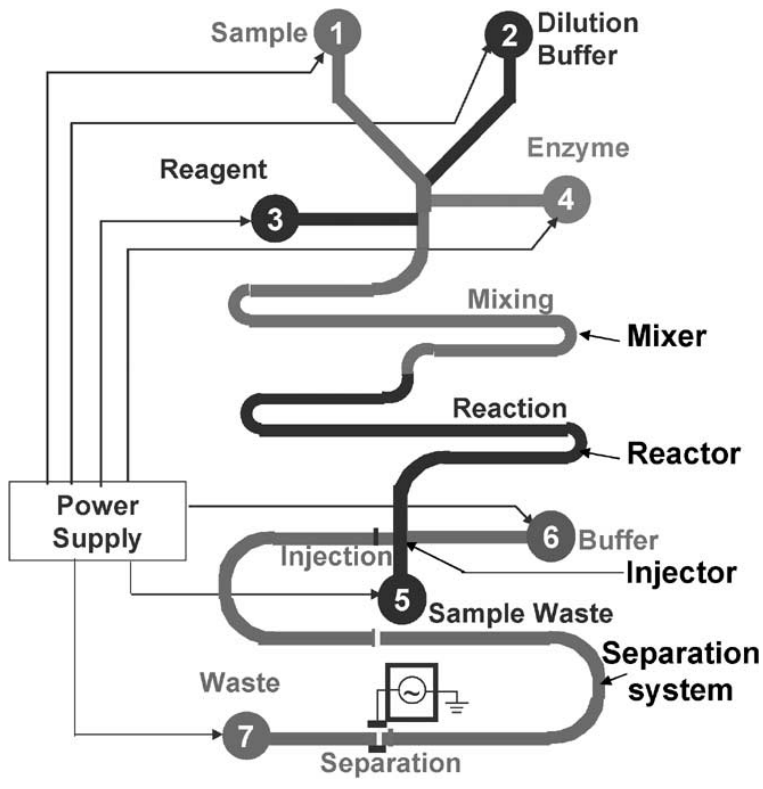

Fig. 2. Sketch of a canonical EK biofluidic LoC. It includes four subsystems of mixing, reaction, injection, and separation. The detection is made at the end of the separation system.

products in the mixer and reactor at this phase are in steady state. This completes the synthesis operation.

In the second phase (analysis), the voltages are switched on reservoirs 6 and 7 with the others left floating. Thus, a band of the analyte is injected from the reaction product into the separation channel for further analysis (in addition to the cross injection shown in Fig. 2, other injection schemes are also available [23]). Because the analyte is comprised of biological species/molecules (e.g., deoxyribonucleic acid (DNA) or amino acids) with different charges and sizes, they move at different speeds and eventually can be separated by electrophoresis [24]. In this phase, the species bands broaden due to molecular diffusion and other dispersion sources; therefore, the transient evolution of the band concentrations is of prime importance.

In this paper, we will discuss the behavioral models and schematic-based simulations of micromixers and electrophoretic separators separately, attributed to their distinctly different biofluidic behavior. However, the top-down design methodology based on hierarchical decomposition that is valid to the entire integrated system [25] will be described jointly (Sections II-B and C).

\section{B. System Hierarchy}

The schematic representation of the biofluidic LoC is based on its geometrical and functional hierarchy. A complex system can be decomposed into a set of commonly used elements of simple geometries, each with an associated function (e.g., mixing or separation), such as straight mixing channels or semicircular turn separation channels. This decomposition enables derivation of a closed-form parameterized model. The elements and their models can be reused in a top-down manner to represent various chip designs using different topologies, element sizes, and material properties. 


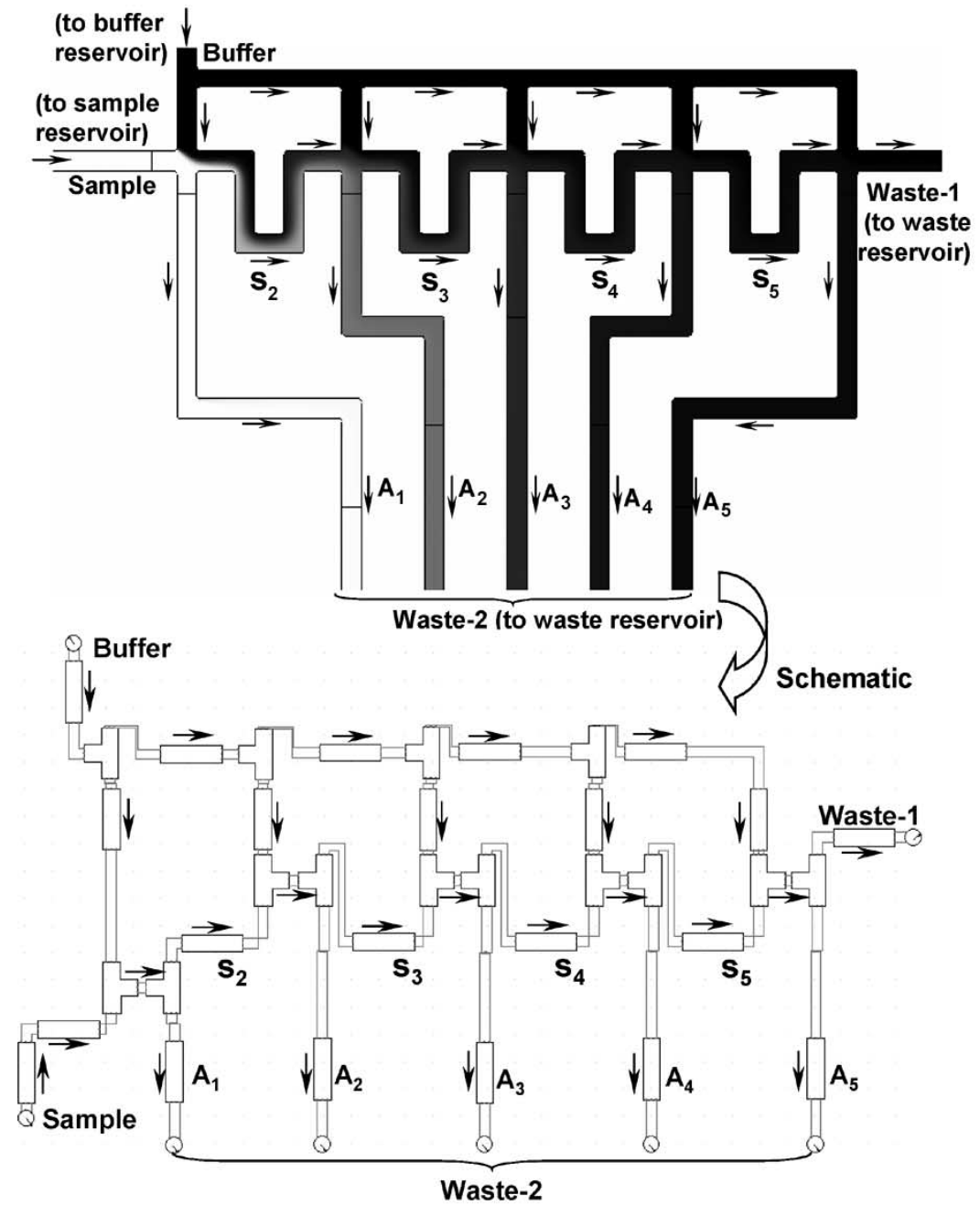

(a)

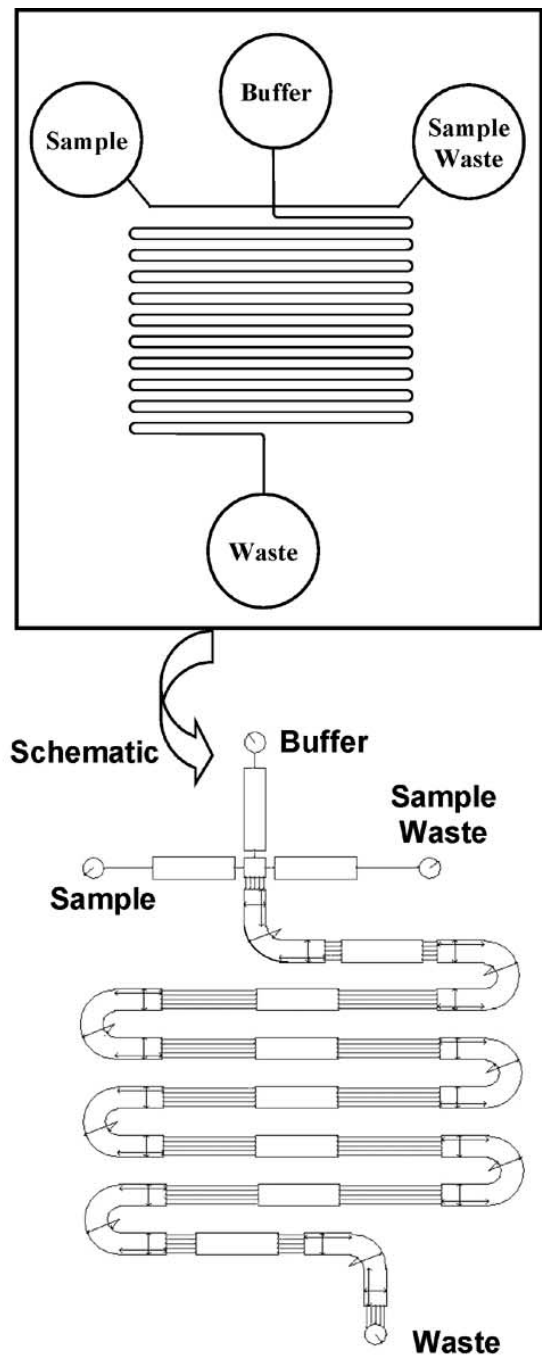

(b)

Fig. 3. (a) EK serial mixing network [3] and its hierarchical schematic representation. The network consists of reservoirs, mixing channels, and T- and crossintersections. Sample and buffer are released and collected by the reservoirs. In the composable approach, the serial mixing network is represented as a collection of interconnected mixing elements composed of microchannels, converging intersections and diverging intersections. (b) A serpentine electrophoretic separation microchip [24] and its schematic representations. Similarly, it is decomposed into a set of elements including reservoirs, injector, straight channels, $180^{\circ}$ turns, and $90^{\circ}$ elbows.

Fig. 3(a) illustrates a complex EK serial mixing network [3] consisting of reservoirs, mixing channels, and T- and crossintersections. The sample is released and collected by the reservoirs at the extreme ends of the mixer. Within the crossintersection, a portion of the input sample is shunted to channels $A_{1}-A_{5}$ and the rest continues along the flow direction for further dilution. Repeating this unit cell in series leads to an array of continuously diluted sample concentrations in channels $A_{1}-A_{5}$, which can be used for parallel biochemical analysis and titration tests. Variations of sample concentrations are represented by grey levels from numerical simulation in Fig. 3(a). In our approach, we represent the serial mixing network as a collection of interconnected mixing elements composed of microchannels, converging intersections, and diverging intersections (note that the double-input and double-output crossintersection is modeled as a combination of the converging and diverging intersections). Fig. 3(b) shows a serpentine electrophoretic separation microchip, which is similarly decomposed into a set of elements including reservoirs, injector, straight channels, $180^{\circ}$ turns, and $90^{\circ}$ elbows. These elements are then wired to form a complete schematic representing the entire LoC according to its chip topology.

\section{Biofluidic Pins and Analog Wiring Buses}

In the schematic, element terminals are connected by groups of pins. Each pin defines the state of biofluidic signal at the element terminals. Pins of the adjacent elements are then linked by wires to enable signal transmission in the hierarchical LoC schematic. Therefore, pin definition affects both schematic composition and behavioral modeling of the elements. There are two types of pins defined in the network. One is the electrical pin at the element terminals. This type of pin is independent of the functions achieved by the $\mathrm{LoC}$ and is present in all elements. It is used to construct a Kirchhoffian network, with both the voltage at the pin and the current flowing through the element. The second type of pin captures the biofluidic state, which is calculated in terms of a directional signal flow 


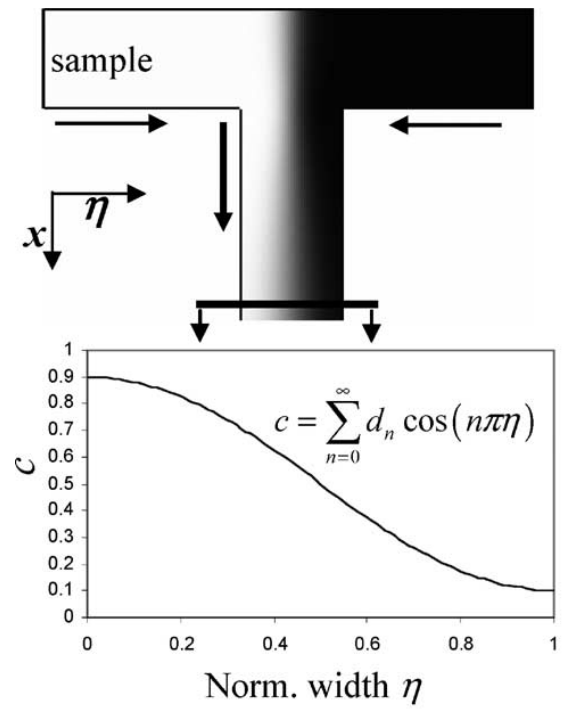

(a)

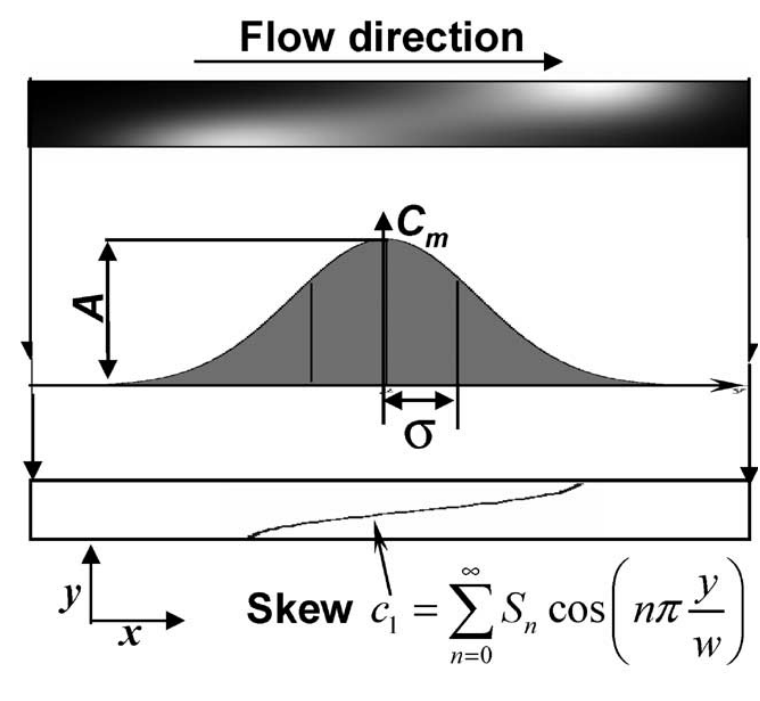

(b)

Fig. 4. (a) Biofluidic pin definition for a micromixer. The pin is defined to convey the concentration coefficients-cosine Fourier series coefficients $\left(d_{n}\right)$ of a

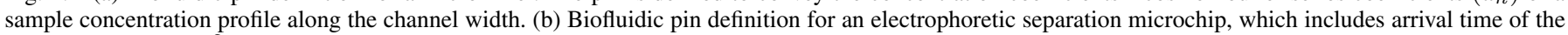
band $(t)$, variance $\left(\sigma^{2}\right)$, skew coefficients $\left(S_{n}\right)$ as well as the amplitude $(A)$.

from the upstream to the downstream. That is, their values at an element outlet are determined from the values at the inlet and the element's own contribution. Pin values at the outlet are assigned to those at the inlet of the next downstream element. Schematic simulation can then serially process each element, starting from the most upstream element. The details of the information that need to be captured for complete definition of the biofluidic state depend on the functionality of the network. In this paper, we focus on two types of biofluidic networks, namely, micromixing and electrophoretic separation.

Within the micromixer, different samples or reagents carried by EK flow mix with each other and their concentrations stay steady state, provided there is a continuous supply from the inlet reservoirs. The sample concentration profile $c$ (as a function of the widthwise position of the channel) describes the biofluidic state at the element terminals in this network, as shown in Fig. 4(a), where $\eta=y / w(0 \leq \eta \leq 1)$ is the normalized widthwise coordinate of mixing elements. Therefore, this pin uses a vector of concentration coefficients $\left\{d_{n}\right\}$, the Fourier cosine series coefficients of the widthwise concentration profile. The reason for such a choice is attributed to the fact that the Fourier cosine series is the eigenfunction of the convection-diffusion equation governing the sample concentration in the network, given insulation condition at channel walls and normalized widthwise position from 0 to 1 .

For the electrophoretic separation system, the injected species bands move through the microchannel accompanied by the band-spreading effect that is caused by dispersions (e.g., molecular diffusion and turn-geometry-induced dispersion [10]). This band spreading adversely affects separation performance by reducing the detectability and separation resolution of the bands. Therefore, the state associated with the species band shape, such as the width of the band, skew, and amplitude as shown in Fig. 4(b), is needed, as well as the time at which the band reaches the element terminals. Specifically, the concentration profile $c$ of a skewed band is first crosssectionally averaged, yielding a distribution of the average concentration $c_{m}$ in the EK flow direction. Thus, pins are defined in terms of the variance $\sigma^{2}$, the square of the standard deviation of $c_{m}$ distribution in the flow direction, representing the width of the band; the Fourier cosine series coefficients $\left\{S_{n}\right\}$ used to reconstruct the skew $c_{1}$ (the centroid positions of the axial filaments of the species band [10], see the Appendix) caused by the nonuniform electric field and migration distance in turns (the Fourier cosine series is used again for the same reason as the above); the separation time $(t)$, the moment the band's centroid reaches the element terminals; and amplitude $(A)$, the maximum average concentration.

The concentration profile of samples in the micromixers and concentration skew of species bands in the separation channels are defined in terms of a vector of Fourier coefficients. For most biofluidic applications, ten terms $(n=1,3, \ldots, 19$ for separation and $n=0, \ldots, 9$ for mixing) for each species/sample are found to yield sufficient computational accuracy due to the quick convergence of the Fourier series. These behavioral models allow for a virtually arbitrary number of different species/samples coexisting in the buffer. Each species requires its own set of pins for the biofluidic state (electrical pins can be shared among species). To reduce the wiring effort between elements, analog wiring buses are employed and the wires connecting the pins of the same discipline are grouped, resulting in only one bus (concentration coefficients) and four buses (separation time, variance, skew, and amplitude) at the terminals of the mixing and separation elements, respectively, as shown in Figs. 5 and 6. Table I summarizes the numbering and disciplines of the buses used in both mixing and separation behavioral models for implementation of three samples/species. Fig. 3 also illustrates the schematics with the symbol view of the behavioral models interconnected by wiring buses. 


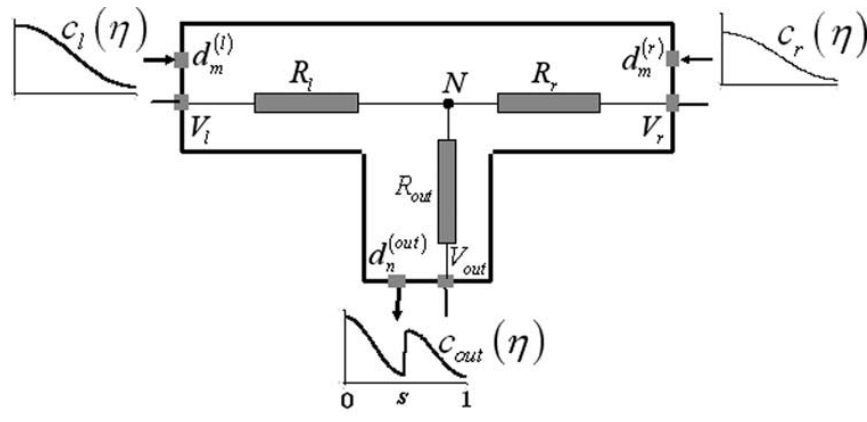

(a)

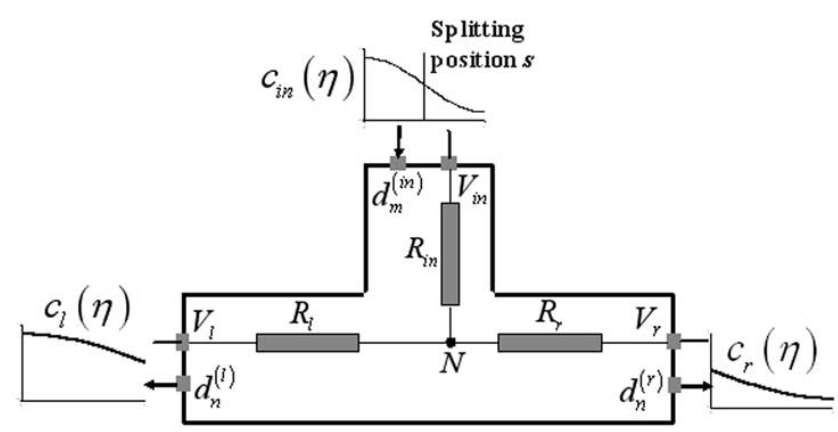

(b)

Fig. 5. (a) Behavioral model structure for the converging intersection in the micromixer. Indexes $l, r$, and out represent the quantities at the left inlet, right inlet, and outlet, respectively. Both electric $(V)$ and biofluidic $\left(d_{m}\right)$ pins are defined at the terminals of the model. Electrically, it is modeled as a combination of three resistors $\left(R_{1}, R_{\mathrm{r}}\right.$, and $\left.R_{\text {out }}\right)$ with zero resistance. Different sample concentration profiles, $c_{1}(\eta)$ and $c_{\mathrm{r}}(\eta)$, at inlets are merged and compressed at the outlet $c_{\text {out }}(\eta)$. (b) Behavioral model structure for the diverging intersection in the micromixer. Similarly, index $l, r$, and in represent the quantities at the left outlet, right outlet, and inlet, respectively. Sample concentration profile at inlet $c_{\text {in }}(\eta)$ is split and stretched out into two parts, $c_{1}(\eta)$ and $c_{\mathrm{r}}(\eta)$, flowing out of both outlets.

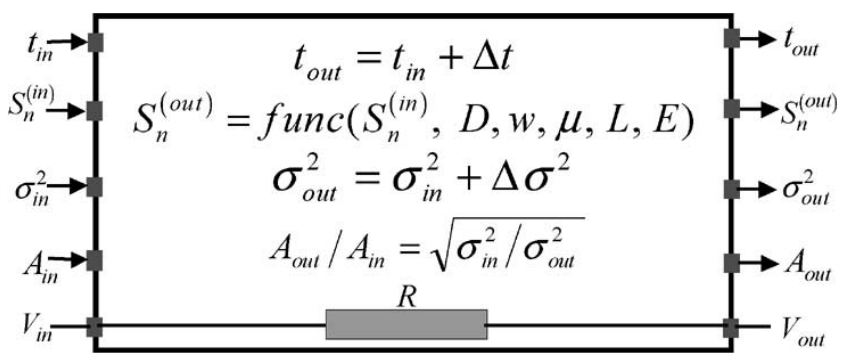

Fig. 6. Behavioral model structure for separation channels in electrophoretic separation microchips. Indexes in and out represent the quantities at the inlet and outlet. Both electric $(V)$ and biofluidic $\left(t, S_{n}, \sigma^{2}\right.$, and $\left.A\right)$ pins are defined at the terminals of the model. Electrically, the channel is modeled as a resistor $(R)$. The variations of biofluidic pin values due to dispersion effects are captured by the model.

\section{Behavioral Models}

The goal of each behavioral model is to capture the inputoutput signal flow relationship of the pin values that define biofluidic state at the inlet and outlet of each element. This captures the physical phenomena being modeled in that element. In addition, an electrical resistance is associated with each element to relate the EK current flow through the element to the inlet and outlet voltages. In contrast to the bottomup reduced-order model approaches, our behavioral models possess several important attributes to enable accurate and efficient system-level simulations of complex LoCs. Our analytical models effectively account for the same multiphysics (e.g., electrostatics, fluidics, and mass transfer) as numerical simulation tools. They do not require any parameters from userconducted experiments or numerical simulations to capture interactions between the elements, and, hence, provide seamless model interconnectivity. Most importantly, they are in closed form and are all parameterized by element dimensions and material properties; therefore, they are reusable, fast to evaluate, and well suited for an iterative simulation-based design methodology.

As discussed above, depending on the physical phenomena of individual devices, contents of the behavioral model libraries will be different. Hence, models for the micromixer and electrophoretic separation system will be developed separately, and are available in separate model libraries for schematic-based simulations.

\section{A. EK Passive Micromixers}

The EK passive micromixer library consists of models for nine elements, which includes reservoirs (sample and waste), slightly tapered straight mixing channel, turns $\left(90^{\circ}\right.$ or $180^{\circ}$, clockwise or counterclockwise), as well as converging and diverging intersections. In this section, we will present behavioral models for basic elements such as the slightly tapered mixing channel and converging and diverging intersections. Other elements can be modeled in a similar fashion.

1) Slightly Tapered Straight Mixing Channels: The tapered straight mixing channel, in which different samples $a$ and $b$ mix with each other, has one inlet and one outlet, with different cross-sectional area. It is critical in designing a geometrical focusing micromixer [26]. Electrically, it is modeled as a resistor, and the resistance is given by

$$
R=\int_{0}^{L} \frac{d z}{w(z) h(z) C_{e}}
$$

where $w$ and $h$ are the channel width and depth (both are functions of the axial coordinate $z$ ) and $C_{e}$ is the electric conductivity of the buffer solution in the channel. As a special case, in a straight channel with the uniform cross section, (1) can be reduced to

$$
R=\frac{L}{h w C_{e}} .
$$

To obtain the sample concentration profile at the outlet, we partition the slightly tapered straight channel into a series of segments (segment number tends to infinity), each with uniform cross section. In each segment, the convection-diffusion equation is solved to establish the input-output relationship of concentration coefficients between the segment terminals. Then all the segmental solutions are multiplied and the concentration coefficients $d_{n}^{\text {(out) }}(n=0,1,2, \ldots)$ at the channel outlet are attained as [27]

$$
d_{n}^{(\text {out })}=d_{n}^{(\mathrm{in})} e^{-\gamma n^{2} \pi^{2} \frac{L D}{E_{\mathrm{in}} \mu w_{\mathrm{in}}^{2}}}
$$


TABLE I

DEFINITION OF BIOFLUIDIC PINS

\begin{tabular}{|c|c|c|}
\hline \multicolumn{3}{|c|}{ Micromixing } \\
\hline Bus & Pins connected & Description \\
\hline$d[0: 29]$ & $\begin{array}{l}\text { Concentration } \\
\text { coefficients }\end{array}$ & $\begin{array}{c}d[0: 9]: \text { the } 1^{\text {st }} \text { sample, } \\
d[10: 19] \text { : the } 2^{\text {nd }}, d[20: 29] \text { : the } 3^{\text {rd }}\end{array}$ \\
\hline \multicolumn{3}{|c|}{ Electrophoretic Separation } \\
\hline Bus & Pins connected & Description \\
\hline$t[0: 2]$ & Separation time & $t[0]$ for the $1^{\text {st }}$ species, $t[1]$ the $2^{\text {nd }}, t[2]$ the $3^{\text {rd }}$ \\
\hline$\sigma^{2}[0: 2]$ & Variance & $\sigma^{2}[0]$ for the $1^{\text {st }}$ species, $\sigma^{2}[1]$ the $2^{\text {nd }}, \sigma^{2}[2]$ the $3^{\text {rd }}$ \\
\hline$A[0: 2]$ & Amplitude & $A[0]$ for the $1^{\text {st }}$ species, $A[1]$ the $2^{\text {nd }}, A[2]$ the $3^{\text {rd }}$ \\
\hline$S[0: 30]$ & Skew coefficients & $\begin{array}{c}S[0] \text { : the direction of the skew caused by the } 1^{\text {st }} \text { turn } \\
S[1: 10]: \text { the } 1^{\text {st }} \text { species } \\
S[11: 20]: \text { the } 2^{\text {nd }}, S[21: 30]: \text { the } 3^{\text {rd }}\end{array}$ \\
\hline
\end{tabular}

where $d_{n}^{(\text {in })}, w_{\text {in }}$, and $E_{\text {in }}$ are the concentration coefficients and the channel width and electric field at the inlet, respectively, $\gamma$ is a factor capturing the effect of the cross-sectional shape on mixing [27], $D$ and $\mu$ are the diffusivity and EK (including both electroosmotic and electrophoretic) mobility of the sample, and $L$ is the channel length. The special case of a straight channel with the uniform cross section yields $\gamma=1$.

2) Converging Intersections: Fig. 5 shows the behavioral model structure of converging and diverging intersections used in micromixers [3]. Arrows at pins indicate the direction of signal flow for computing biofluidic pin values and state. The converging intersection has two inlets and one outlet, and acts as a combiner to align and compress upstream sample flows of an arbitrary flow ratio $s$ (defined below) and concentration profiles side by side at its outlet [Fig. 5(a)]. As its flow path lengths are negligibly small compared with those of mixing channels, such an element can be assumed to have zero physical size, and electrically represented as three resistors with zero resistance between each terminal and the internal node $N$

$$
R_{\mathrm{l}}=R_{\mathrm{r}}=R_{\text {out }}=0 .
$$

Here, $N$ corresponds to the intersection of flow paths and subscripts $l, r$, and out represent the left and right inlets, and the outlet, respectively. Defining $d_{m}^{(1)}$ and $d_{m}^{(\mathrm{r})}(m=0,1,2 \ldots)$ as the Fourier coefficients of the sample concentration profiles at the left and right inlets, respectively. Then the coefficients $d_{n}^{\text {(out) }}(n=0,1,2 \ldots)$ of the profile at the outlet $\left(c_{\text {out }}(\eta)\right)$ are related to $d_{m}^{(1)}$ and $d_{m}^{(\mathrm{r})}$ by

$$
\begin{aligned}
c_{\mathrm{out}}(\eta) & =\sum_{n=0}^{\infty} d_{n}^{\text {(out) }} \cos (n \pi \eta) \\
& = \begin{cases}\sum_{m=0}^{\infty} d_{m}^{(1)} \cos \left(\frac{m \pi \eta}{s}\right), & 0 \leq \eta<s \\
\sum_{m=0}^{\infty} d_{m}^{(\mathrm{r})} \cos \left(\frac{m \pi(\eta-s)}{1-s}\right), & s \leq \eta<1 .\end{cases}
\end{aligned}
$$

Equation (5) shows that the concentration profile at the outlet can be treated as a superposition of the scaled-down profiles from both inlets, where $s=q_{1} /\left(q_{1}+q_{\mathrm{r}}\right)=I_{1} /\left(I_{1}+I_{\mathrm{r}}\right)$ denotes the interface position [or flow ratio, the ratio of the left flow rate $q_{1}$ to the total flow rate $\left(q_{1}+q_{\mathrm{r}}\right)$ ] between incoming streams in the normalized coordinate at the outlet (note that flow rates $q_{\mathrm{l}}$ and $q_{\mathrm{r}}$ are, respectively, linear with the electric currents $I_{1}$ and $I_{\mathrm{r}}$ ).

Solving (5) yields $d_{n}^{\text {(out) }}$, written as shown in (6) at the bottom of the page, where $f_{1}=(m-n s) \pi, f_{2}=(m+n s) \pi$, $F_{1}=(m+n-n s) \pi$, and $F_{2}=(m-n+n s) \pi$. Since the sample concentration profiles at the inlets are scaled down, the Fourier series modes at the inlets are not orthogonal to those at the outlet. Therefore, the calculation of the coefficient for a certain Fourier mode at the outlet depends on all the modes at the inlets.

$$
\left\{\begin{aligned}
d_{0}^{(\text {out })}= & d_{0}^{(\mathrm{l})} s+d_{0}^{(\mathrm{r})}(1-s) \\
d_{n>0}^{(\text {out })}= & s \sum_{m=0}^{\infty, \text { if } m \neq n s} d_{m}^{(1)} \frac{f_{1} \sin \left(f_{2}\right)+f_{2} \sin \left(f_{1}\right)}{f_{1} f_{2}}+s \sum_{m=0}^{\infty, \text { if } m=n s} d_{m}^{(1)}+(1-s) \sum_{m=0}^{\infty, \text { if }}(-1)^{n-m} d_{m}^{(\mathrm{r})} \\
& +2(-1)^{n}(1-s) \sum_{m=0}^{\infty, \text { if }} \sum_{m \neq n(1-s)} d_{m}^{(\mathrm{r})}\left(\frac{\cos \left(\frac{F_{2}}{2}\right) \sin \left(\frac{F_{1}}{2}\right)}{F_{1}}+\frac{\cos \left(\frac{F_{1}}{2}\right) \sin \left(\frac{F_{2}}{2}\right)}{F_{2}}\right)
\end{aligned}\right.
$$


3) Diverging Intersections: The diverging intersection has one inlet and two outlets and is the dual of the converging intersection. It splits the incoming flow and electric current into two streams that exit out of the outlets. It can also be represented by three zero-resistance resistors

$$
R_{\text {in }}=R_{\mathrm{l}}=R_{\mathrm{r}}=0
$$

where subscripts in, 1 , and $\mathrm{r}$ represent quantities at the inlet and the left and right outlets, respectively.

Defining $d_{m}^{(\text {in })}(m=0,1,2, \ldots)$ as the Fourier coefficients of the sample concentration profile at the inlet. Then the coefficients at the left and right outlets $d_{n}^{(1)}$ and $d_{n}^{(\mathrm{r})}$ are, respectively, given by

$$
\left\{\begin{aligned}
d_{0}^{(1)}= & d_{0}^{(\text {in })}+\sum_{m=1}^{\infty} \frac{d_{m}^{(\text {in })} \sin \left(\phi_{1}\right)}{\phi_{1}} \\
d_{n>0}^{(1)}=2 & \sum_{m=0}^{\infty, \text { if } m \neq \frac{n}{s}} \frac{d_{m}^{(\text {in })}(-1)^{n+1} \phi_{1} \sin \left(\phi_{1}\right)}{f_{1} f_{2}} \\
& +\sum_{m=0}^{\infty, \text { if } m=\frac{n}{s}} d_{m}^{(\text {in })}
\end{aligned}\right.
$$

and

$$
\left\{\begin{array}{c}
d_{0}^{(\mathrm{r})}=d_{0}^{(\mathrm{in})}-\sum_{m=1}^{\infty} \frac{d_{m}^{(\mathrm{in})} \sin \left(\phi_{1}\right)}{\phi_{2}} \\
d_{n>0}^{(\mathrm{r})}=2 \sum_{m=0}^{\infty, \text { if }} \frac{n}{m \neq \frac{n}{(1-s)}} \frac{d_{m}^{(\mathrm{in})} \phi_{2} \sin \left(\phi_{1}\right)}{F_{1} F_{2}} \\
+\sum_{m=0}^{\infty, \text { if }} m=\frac{n}{(1-s)}(-1)^{m-n} d_{m}^{(\mathrm{in})}
\end{array}\right.
$$

where $f_{1}=(n-m s) \pi, f_{2}=(n+m s) \pi, \quad F_{1}=(n+m-$ $m s) \pi, F_{2}=(n-m+m s) \pi, \phi_{1}=m s \pi$, and $\phi_{2}=m(1-s) \pi$. Similar to the converging intersection, $s$ is the normalized splitting position (or ratio).

It should be pointed out that in contrast to the resistor-based mixing models [3], [17] that take advantage of the analogy between fluidic and sample transports and only convey the average concentration values through the entire network, our models [(3), (6), (8), and (9)] propagate sample concentration profiles characterized by the Fourier series coefficients. This removes the requirement of complete mixing (along channel width) at the end of each channel [3] in the network imposed by the resistor-based models and allows for optimal design of both effective and efficient micromixers.

\section{B. Electrophoretic Separation Chips}

The electrophoretic separation library includes models for ten basic elements: turns $\left(90^{\circ}\right.$ or $180^{\circ}$, clockwise or counterclockwise), straight channel, detector, injector, injection channel, and reservoirs (sample and waste). In this section, behavioral models for basic elements such as separation chan- nels (straight and turn) will be developed to analyze the band-spreading effect caused by molecular diffusion and turn dispersion. Additionally, a detector model applicable for both direct current (dc) and transient analysis will be presented. Models of the other elements can be derived using the same principles.

Fig. 6 shows the behavioral model structure of electrophoretic separation channels (straight or turn). Arrows indicate the direction of signal flow for calculating biofluidic pin values and state. Electrically, separation channels are modeled as resistors in the same way as the uniform straight mixing channels (for a constant-radius turn, $L$ in (2) is replaced by $L=r_{c} \theta$, where $r_{c}$ and $\theta$ are the mean radius and angle included by the turn, see [10] and [28] for the detailed geometrical interpretation). Additionally, symbols and characters used in this section are defined the same as those for the mixer, unless otherwise noted. The residence time $\Delta t$ of a species band within a separation channel (the time for the band's centroid to move from the channel inlet to outlet) is given by

$$
\Delta t=\frac{L}{\mu E}
$$

The calculation of changes in the skew coefficients and variance depends on the specific element [10] and the inherent variable is the residence time $\Delta t$ obtained by (10). For a straight separation channel

$$
\begin{aligned}
S_{n}^{(\text {out })} & =S_{n}^{(\mathrm{in})} e^{-(n \pi)^{2} \frac{\Delta t D}{w^{2}}} \\
\sigma_{\text {out }}^{2}-\sigma_{\text {in }}^{2} & =\Delta \sigma^{2}=2 D \Delta t .
\end{aligned}
$$

For a separation turn

$$
\begin{aligned}
& S_{n}^{\text {(out) }} \\
& = \pm \frac{8 \theta w^{2}\left(1-e^{-(n \pi)^{2} \frac{\Delta t D}{w^{2}}}\right)}{(n \pi)^{4} \Delta t D}+S_{n}^{(\mathrm{in})} e^{-(n \pi)^{2} \frac{\Delta t D}{w^{2}}} \\
& \quad n=1,3,5 \ldots \\
& \sigma_{\text {out }}^{2}-\sigma_{\mathrm{in}}^{2} \\
& =\Delta \sigma^{2} \\
& =2 D \Delta t \pm \frac{8 w^{4} \theta}{D \Delta t} \sum_{n=1,3,5 \ldots}^{\infty}\left(\frac{S_{n}^{(\mathrm{in})}\left(1-e^{-(n \pi)^{2} \frac{D \Delta t}{w^{2}}}\right)}{(n \pi)^{4}}\right) \\
& \quad+\frac{64 w^{6} \theta^{2}}{(D \Delta t)^{2}} \sum_{n=1,3,5 \ldots}^{\infty} \frac{\left(-1+e^{-(n \pi)^{2} \frac{D \Delta t}{w^{2}}+(n \pi)^{2} \frac{D \Delta t}{w^{2}}}\right)}{(n \pi)^{8}}
\end{aligned}
$$

where subscripts/superscripts in and out represent quantities at the inlet and outlet of the channel, respectively. In (13) and (14), the "+" sign is assigned to the first turn, and any turn strengthening the skew caused by the first; the "-" sign is assigned to any turn undoing the skew from the first. For example, in Fig. 3(b), the first $90^{\circ}$ elbow and the three $180^{\circ}$ turns on the left are all given a "+" sign, in which the species 
band flows counterclockwise. On the contrary, the three $180^{\circ}$ turns on the right use a "-" sign, in which the band migrates clockwise.

Assuming a Gaussian distribution of the average concentration $c_{m}$ of the species band at element terminals, we can obtain the amplitude of the species band by

$$
\frac{A_{\text {out }}}{A_{\text {in }}}=\sqrt{\frac{\sigma_{\text {in }}^{2}}{\sigma_{\text {out }}^{2}}} .
$$

For the detector model, the variance change associated with the detector path length $L_{\text {det }}$ is given by [24]

$$
\Delta \sigma^{2}=\frac{L_{\mathrm{det}}^{2}}{12}
$$

\section{Model Implementation}

To demonstrate use of the above parameterized models for top-down designs, we have implemented the models in the Verilog-A analog hardware description language. Symbol view for each of the elements is used to compose a schematic within Cadence's [29] integrated circuit design framework (e.g., Fig. 3). The Cadence design framework is used to automatically netlist the complex topologies in the biofluidic LoC schematics, and Spectre is used as the simulator. Similar tools from other vendors, or custom schematic entry tools and solvers that can handle both signal flow and Kirchhoffian networks, could have been also used.

An important issue of implementing separation channel models of turn geometry [(13) and (14)] is the real-time determination of the turn "sign". Providing this flexibility allows a single turn symbol to be reused for constructing arbitrary topologies such as a serpentine, spiral, or their combination thereof, as will be shown later. To address this, two sets of flags are used in the models. One is the system flag $F_{\mathrm{s}}$, stored as the zeroth component of the skew coefficients $(S[0]$ in Table I) to record the direction of the skew caused by the first turn or elbow. The other is the intrinsic flag $F_{\mathrm{i}}$ of individual elements. For example, $F_{\mathrm{i}}=1$ is for turns or elbows involving clockwise flow of species bands; $F_{\mathrm{i}}=2$ is for counterclockwise turns or elbows. Since straight channels do not incur any skew, no flag is needed. During simulations, $F_{\mathrm{s}}=0$ (i.e., $S[0]=0$ ) is first generated by the injector, which is the most upstream element of a separation channel and, hence, initiates the computation of the separation state. Then as the species band migrates to the first turn or elbow, $F_{\mathrm{s}}$ is irreversibly set to its intrinsic flag $F_{\mathrm{i}}$. Afterward, the written $F_{\mathrm{s}}$ is compared with $F_{\mathrm{i}}$ of each downstream element as the band moves on. If they are identical, a "+" sign is used for the element, otherwise, a "-" sign.

Fig. 7 shows the codes for a $180^{\circ}$ turn involving clockwise flow of species bands to implement this logic and determine the sign.

\section{SChEMATIC-BASEd Simulations}

In this section, we will first describe the simulation procedure, in which the Kirchhoff's resistor network to predict electric current and field as well as the signal flow network to evaluate biofluidic state values (e.g., steady-state mixing concentrations and transient electrophoretic species band shapes) are solved sequentially. Then, the results of schematic simulations exploring various micromixers and separation microchips will be discussed and validated with numerical and experimental data.

\section{A. Simulation Description}

Schematic simulations for mixers and separation chips involve both electric and biofluidic calculations. For dc analysis, given the applied potential at reservoirs, system topology and element dimensions, nodal voltages at element terminals within the entire system are first computed by Ohm's and Kirchhoff's laws using the resistor models presented in the last section. The resulting nodal voltages and branch currents are, in turn, used to calculate the electric field strength $(E)$ and its direction within each element, as well as flow and splitting ratios at intersections (for mixers). With these results and user-provided sample properties $(D$ and $\mu$ ), the sample speed is then given by $u=\mu E$. Next, values of biofluidic pins at the outlet(s) of each element (e.g., concentration coefficients for micromixers; arrival time, variance, skew, and amplitude for separation microchips) are determined. The process starts from the most upstream element, typically the sample reservoirs in mixers and the injector in separation chips in terms of the directional signal flow as described in Section III. As such, both electric and fluidic information in the entire system is obtained.

As described in Section II-A, the mixer operates in steady state, while transient evolution is critical in separation channels. Transient analysis can also be conducted for separation chips that involve the species band's motion and broadening. An electropherogram (average concentration $c_{m}$ versus time) can be obtained at the detector, yielding an intuitive picture of separation resolution between species bands. The transient analysis first calculates for the dc operating points of the amplitude $A_{\text {det }}$, separation time $t_{\mathrm{det}}$, and variance $\sigma_{\mathrm{det}}^{2}$ of the species band at the detector as described above. Based on these points, the actual read-out time is scanned and the average concentration output $c_{m}$ is calculated. Assuming the species band does not appreciably spread out as it passes through the detector, $c_{m}$ is given by

$$
c_{m}=A_{\operatorname{det}} e^{\frac{-(E \mu)^{2}\left(t-t_{\mathrm{det}}\right)^{2}}{2\left(\sigma_{\mathrm{det}}^{2}+\Delta \sigma^{2}\right)}}
$$

where $t$ is the actual read-out time and $\Delta \sigma^{2}$ is the variance growth associated with detection and given in (16).

\section{B. Results and Discussion}

In this section, simulation examples of complex EK passive mixers and electrophoretic separation microchips will be presented to verify the behavioral models for biofluidic elements and validate the modeling and simulation methodology. Schematic simulations for micromixers are shown in Figs. 8-10 


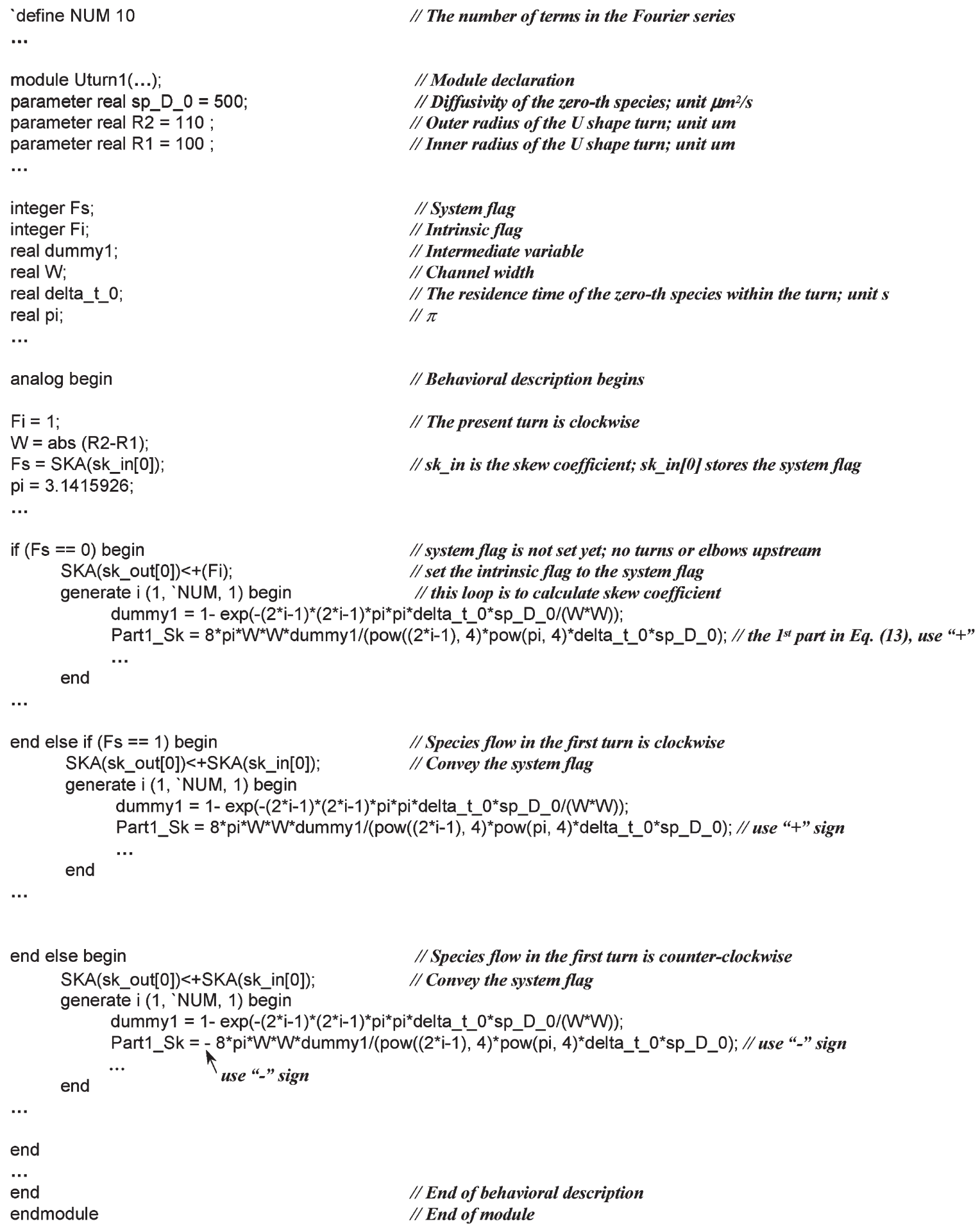

Part1_Sk $=8^{*} \mathrm{pi}^{*} \mathrm{~W}^{*} \mathrm{~W}^{*} \mathrm{dummy} 1 /\left(\operatorname{pow}\left(\left(2^{*} \mathrm{i}-1\right), \overline{4}\right)^{*} \operatorname{pow}(\mathrm{pi}, 4)^{*}\right.$ delta_t_O*sp_D_0 $)$; // use "+" sign

end $\quad$ Part1_Sk $=-8^{*} \mathrm{pi}^{*} \mathrm{~W}^{*} \mathrm{~W}^{*} \mathrm{~d}$

// Module declaration

// Diffusivity of the zero-th species; unit $\mu \mathrm{m}^{2} / \mathrm{s}$

// Outer radius of the $U$ shape turn; unit um

// Inner radius of the $U$ shape turn; unit um

// System flag

// Intrinsic flag

// Intermediate variable

// Channel width

// The residence time of the zero-th species within the turn; unit s

$/ / \pi$

// Behavioral description begins

// The present turn is clockwise

//sk_in is the skew coefficient; sk_in[0] stores the system flag

// system flag is not set yet; no turns or elbows upstream

// set the intrinsic flag to the system flag

// this loop is to calculate skew coefficient

$\cdots$

// Species flow in the first turn is clockwise

// Convey the system flag 


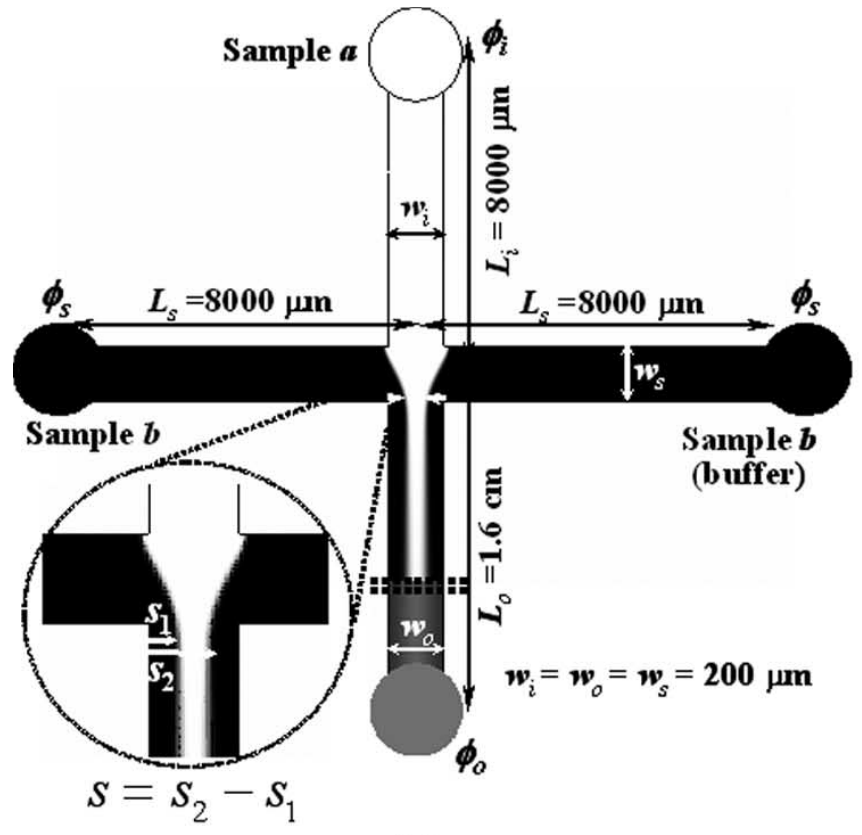

(a)

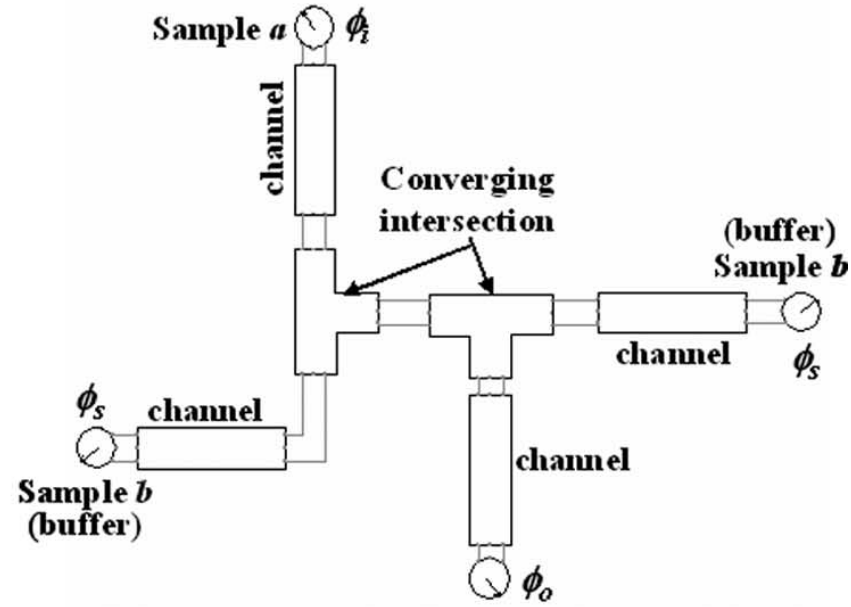

(b)

Fig. 8. (a) EK focusing micromixer. Sample $a$, flowing from the top input channel to the intersection, is pinched by sample $b$ (or buffer) from both side channels. Then samples mix in the bottom mixing channel. (b) Its hierarchical schematic representation. The triple-input and one-output cross-intersection is modeled as a cascade connection of two converging intersections.

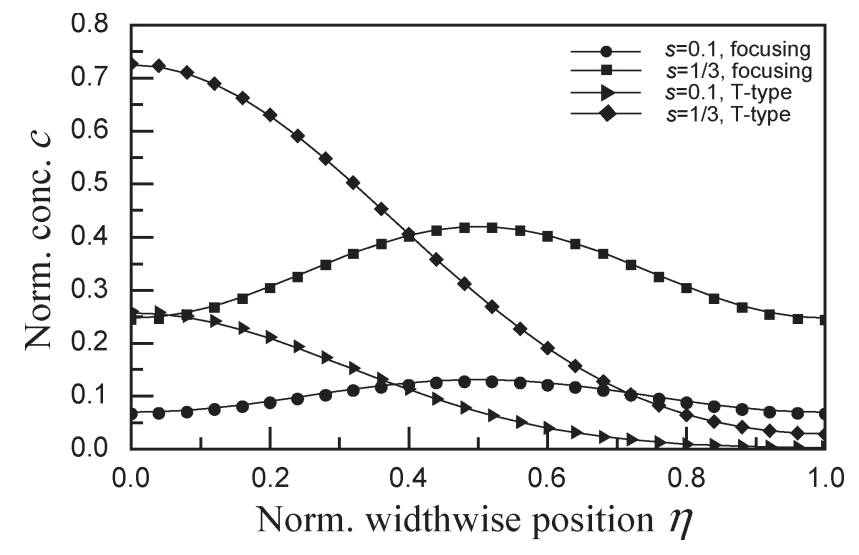

Fig. 9. Schematic simulation results (lines) compared with numerical data (symbols) on concentration profiles $c$ (sample $a$ ) along the normalized channel width $\eta$ for the EK focusing and T-type mixers. In contrast to the T-type mixer, the focusing mixer considerably improves sample homogeneity due to the reduced diffusion distance between samples.

Fig. 9 shows numerical and schematic simulation results of sample $a$ concentration profiles at the mixing channel outlet for two flow ratios $s=0.1$ and $s=1 / 3$. During simulations, reservoir voltages $\left(\phi_{\mathrm{i}}\right.$ and $\left.\phi_{\mathrm{s}}\right)$ are chosen to vary $s$ while keeping $E(143 \mathrm{~V} / \mathrm{cm})$ and the sample residence time fixed in the mixing channel. Excellent agreement between numerical and schematic simulation results is found with the worst case relative error of 3\% at $s=0.1$. The results are also compared with those from a T-type mixer that has the same electrical field in the mixing channel, channel length, and width as the focusing mixer. The focusing mixer considerably improves sample homogeneity, which can be attributed to the reduced diffusion distance between samples. That is, the axial centerline of the mixing channel in the focusing mixer can be treated as

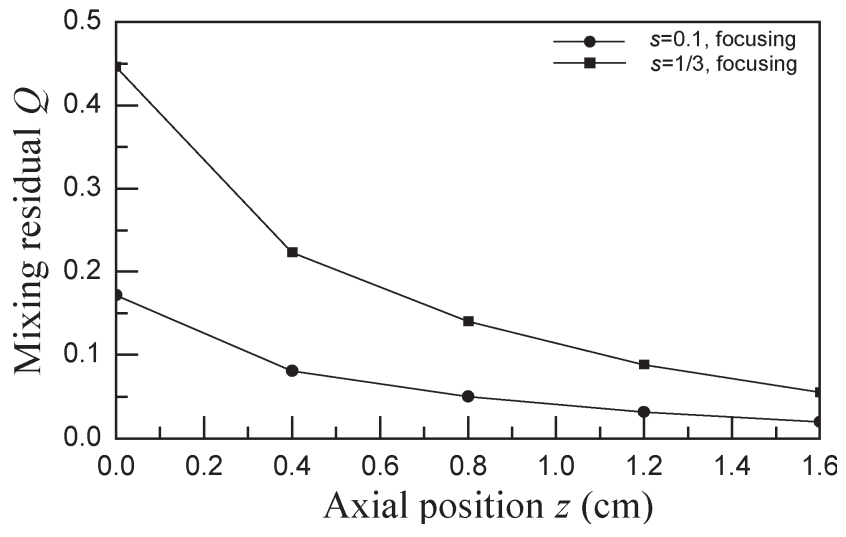

Fig. 10. Schematic simulation results on variation of mixing residual $Q$ along axial channel length (data points are connected by lines to guide the eye) for the EK focusing mixer involving different stream width $s$. A smaller stream width (e.g., $s=0.1$ ) yields a lower initial mixing residual and a more uniform concentration profile at the end of the mixing channel.

an impermeable wall due to symmetry, hence, the diffusion distance is only one-half of that of the T-type mixer. Additionally, a smaller stream width (e.g., $s=0.1$ ) yields a more uniform concentration profile at the end of the mixing channel.

To gain the insight of the influence of the stream width on mixing performance, an index of mixing residual, $Q=$ $\int_{0}^{1}\left|c(\eta)-c_{\text {avg }}\right| d \eta$, is introduced in Fig. 10 to characterize the nonuniformity of concentration profiles, where $c(\eta)$ and $c_{\text {avg }}$ are the normalized concentration profile and widthaveraged concentration, respectively, at the detection spot. At the channel inlet $(z=0)$, mixing residual $Q$ strongly depends on $s$. Asymmetric incoming streams yield a lower $Q$ value (e.g., $Q=0.18$ at $s=0.1$ compared with $Q=0.44$ at $s=1 / 3$ ) and a more uniform initial profile. Along the channel, 
TABLE II

COMPARISON OF SCHEMATIC SimUlation RESUlts (sche) With NuMERICAL (num) AND EXPERIMENTAL (exp) DATA ON Sample Concentrations in Analysis Channels of Serial and Parallel Mixing Networks [3]

\begin{tabular}{|c|c|c|c|c|c|c|c|c|c|}
\hline \multicolumn{6}{|c|}{ Serial Mixing Network } & \multirow{2}{*}{\multicolumn{4}{|c|}{$\begin{array}{r}\text { Parallel Mixing Network } \\
\text { Complete Mixing }\end{array}$}} \\
\hline & \multicolumn{3}{|c|}{ Complete Mixing } & \multicolumn{2}{|c|}{ Partial Mixing } & & & & \\
\hline channel & $c$ (sche) & $c(\exp )$ & $c$ (num) & $c$ (sche) & $c$ (num) & channel & $c$ (sche) & $c(\exp )$ & $c$ (num) \\
\hline $\mathrm{A}_{1}$ & 1 & 1 & 1 & 1 & 1 & $\mathrm{~A}_{1}$ & 0 & 0 & 0 \\
\hline $\mathrm{A}_{2}$ & 0.37 & 0.36 & 0.378 & 0.48 & 0.496 & $\mathrm{~A}_{2}$ & 0.83 & 0.84 & 0.832 \\
\hline $\mathrm{A}_{3}$ & 0.22 & 0.21 & 0.224 & 0.187 & 0.187 & $\mathrm{~A}_{3}$ & 0.68 & 0.67 & 0.674 \\
\hline $\mathrm{A}_{4}$ & 0.125 & 0.13 & 0.133 & 0.081 & 0.0815 & $\mathrm{~A}_{4}$ & 0.52 & 0.51 & 0.523 \\
\hline \multirow[t]{3}{*}{$\mathrm{A}_{5}$} & 0.052 & 0.059 & 0.0628 & 0.029 & 0.0315 & $\mathrm{~A}_{5}$ & 0.35 & 0.36 & 0.354 \\
\hline & & & & & & $\mathrm{A}_{6}$ & 0.17 & 0.19 & 0.168 \\
\hline & & & & & & $\mathrm{A}_{7}$ & 1 & 1 & 1 \\
\hline
\end{tabular}

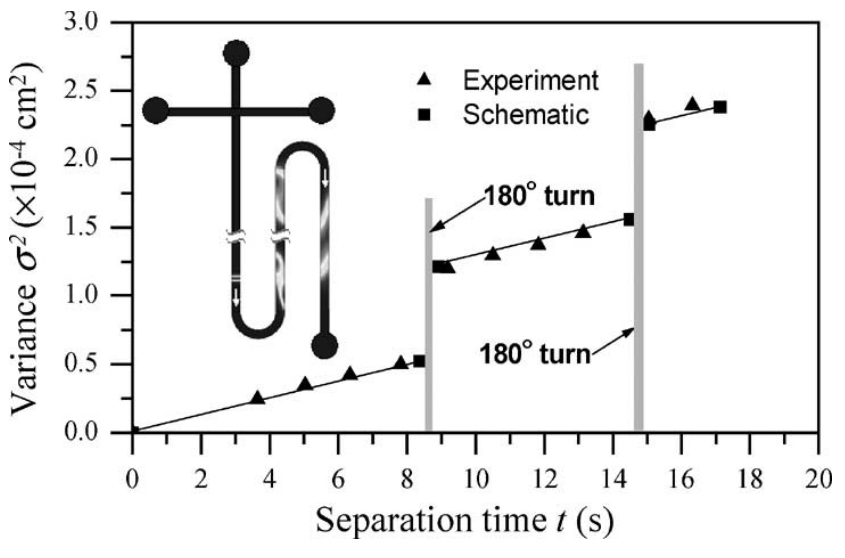

Fig. 11. Comparison of experimental data [6] with de schematic simulation on variance $\sigma^{2}$ versus separation time $t$ of species $a$ in a serpentine electrophoretic separation microchip of two complementary turns. The grey bars represent the residence time of the sample within the turns. The first turn skews the species band and accordingly incurs abrupt increase in variance. The transverse diffusion in the interturn straight channel smears out most of the skew and presents a nearly uniform band before the second turn (see the inset of numerical simulation plot). The second turn then distorts the band again in the opposite direction, leading to another turn-induced variance.

$Q$ initially drops rapidly and then becomes saturated, because the improved sample mixing reduces the concentration gradient and the driving force for further mixing. Thus, a tradeoff between $Q$ and mixer size can be captured by the behavioral models presented in this paper to achieve designs of both effective and efficient micromixers.

These parameterized and reusable behavioral models are well suited to study complex mixing networks [3], in which an array of sample concentrations can be obtained at multiple analysis channels by geometrically duplicating units with a single constant voltage applied at all reservoirs.

Table II shows the comparison of schematic simulation results with experimental and numerical data on sample (rhodamine B) concentrations in analysis channels $A_{1}-A_{5}$ in the serial mixing network [Fig. 3(a)]. Both complete and partial mixing cases are investigated. When a voltage of $0.4 \mathrm{kV}$ is applied at the sample and buffer reservoirs with the waste reservoirs grounded, sample mixing in channels $\mathrm{S}_{2}-\mathrm{S}_{5}$ is widthwisely complete. There is excellent agreement of the schematic simulation results with numerical analysis and experimental data (with an average error smaller than 6\%). In contrast to the electric resistor-based models [3], [17], [31] that take advantage of the analogy between EK flow and electric current and require postcalculations of concentrations from electric currents in the network, our behavioral models directly yield the concentration value in each analysis channel. In addition to complete mixing, partial mixing case is also schematically simulated. A voltage of $1.6 \mathrm{kV}$, as used in the experiments in the literature [3], is applied at the sample and buffer reservoirs with the waste grounded, which increases the EK velocity and then decreases the residence time of the sample by fourfold in channels $\mathrm{S}_{2}-\mathrm{S}_{5}$. Thereby, the mixing in channels $\mathrm{S}_{2}-\mathrm{S}_{5}$ is widthwisely incomplete, and the amount of sample shunted to channels $A_{1}-A_{5}$ depends not only on the electric currents in the network but also on the sample concentration profiles at the exits of channels $\mathrm{S}_{2}-\mathrm{S}_{5}$, which violates the assumption for the analogy between EK flow and sample transport, and, hence, the resistor-based modeling becomes invalid. However, it can be readily simulated by our behavioral models. In the schematic, the cross-intersection is modeled as a combination of the converging and diverging intersections, in which the sample concentration profiles of the incoming and outgoing streams are accurately captured. Results from schematic simulations are compared with numerical data in Table II (a comparison to experimental data is not allowed due to a lack of knowledge of sample properties. Hence, a diffusivity of $D=3 \times 10^{-10} \mathrm{~m}^{2} / \mathrm{s}$ and an EK mobility of $\mu=2.0 \times 10^{-8} \mathrm{~m}^{2} / \mathrm{V} \cdot \mathrm{s}$ are assumed in numerical simulations). Very good agreement can be observed with an average error of $4 \%$. At the cross-intersection following channel $\mathrm{S}_{2}$, the amount of sample shunted to $A_{2}$ is more than that predicted by the complete-mixing case due to the nonuniform sample profiles at the intersection's inlet. Consequently, concentrations in channels $A_{3}-A_{5}$ show the lower values, which agrees with the experimental observations [3]. Netlisting and schematic simulation of this exam ple take $20 \mathrm{~s}$ on a multi-user two-CPU $1-\mathrm{GHz}$ Sun Fire 280 processors with 4-GB random access memory (RAM) for the first-time simulation, and less than a second for subsequent iterations, leading to a 1000-20 000× speedup.

In addition to the serial mixing network, the parallel mixing network [3] can be hierarchically represented and simulated in a similar fashion, and excellent agreement among schematic simulations results, numerical analysis, and experimental data (with an average error of $3.6 \%$ relative to experiments) is also found.

2) Electrophoretic Separation Microchips: Schematic simulation results for electrophoretic separation microchips are shown in Figs. 11-16. In Figs. 11 and 12, a serpentine 


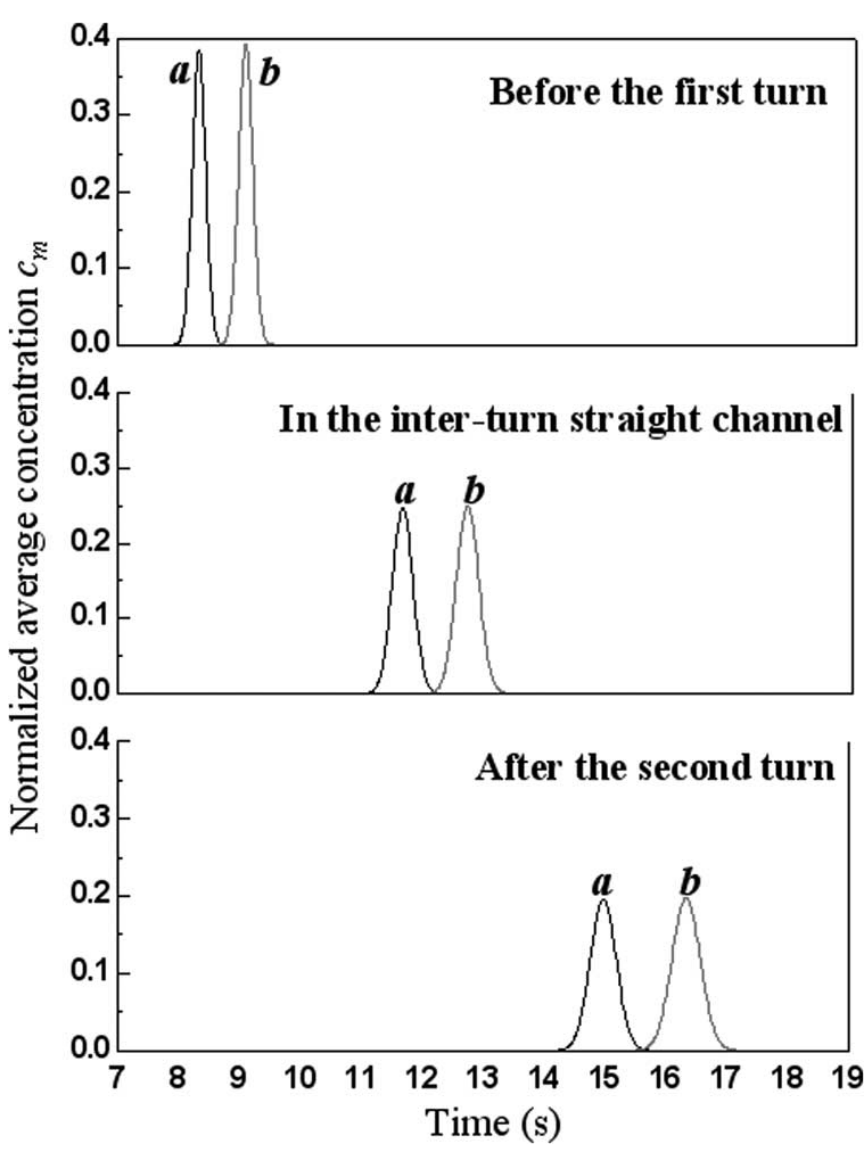

Fig. 12. Transient analysis simulates the electropherograms output from three detectors, which are, respectively, arranged before the first turn (top trace), in the middle of the interturn straight channel (middle trace), and after the second turn (bottom trace). Attributed to the difference in EK mobility, the spacing between concentration peaks of species $a$ and $b$ increases as they migrate through the channels. The dispersion effect leads to the continuous decreases in the band amplitude.

electrophoresis column of two complementary turns is used to separate an analyte comprised of two species $a(D=3.12 \times$ $\left.10^{-10} \mathrm{~m}^{2} / \mathrm{s}, \mu=1.2 \times 10^{-8} \mathrm{~m}^{2} / \mathrm{s} \cdot \mathrm{V}\right)$ and $b(D=2.72 \times$ $\left.10^{-10} \mathrm{~m}^{2} / \mathrm{s}, \mu=1.1 \times 10^{-8} \mathrm{~m}^{2} / \mathrm{s} \cdot \mathrm{V}\right)$ with $E=600 \mathrm{~V} / \mathrm{cm}$. Experimental data [6] on variance versus time of species $a$ are compared with dc schematic simulations in Fig. 11, showing excellent agreement with the worst case relative error of only $5 \%$. Again, netlisting and dc simulation for this example take $20 \mathrm{~s}$ for the first-time iteration and less than a second for subsequent iterations, leading to a 500-10000× speedup (higher speedup can be obtained for a more complex chip topology or a less diffusive species as shown in Fig. 15). The first turn skews the species band and accordingly incurs abrupt increase in variance. During the species band's migration in the long interturn straight channel, the transverse diffusion smears out most of the skew and presents a nearly uniform band before the second turn. The second turn then distorts the band again in the opposite direction, leading to another turn-induced variance that is equal to the one caused by the first turn. Fig. 12 shows separation electropherograms of both species from three detectors. The spacing between concentration peaks of species $a$ and $b$ increases as they migrate through channels, but due to the bandbroadening effect, the amplitude decreases consecutively.

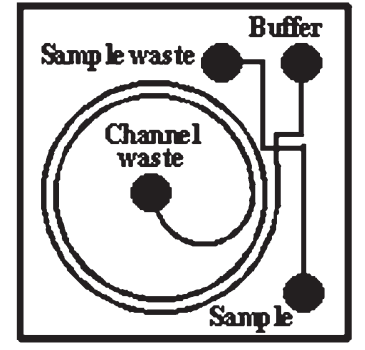

(a)

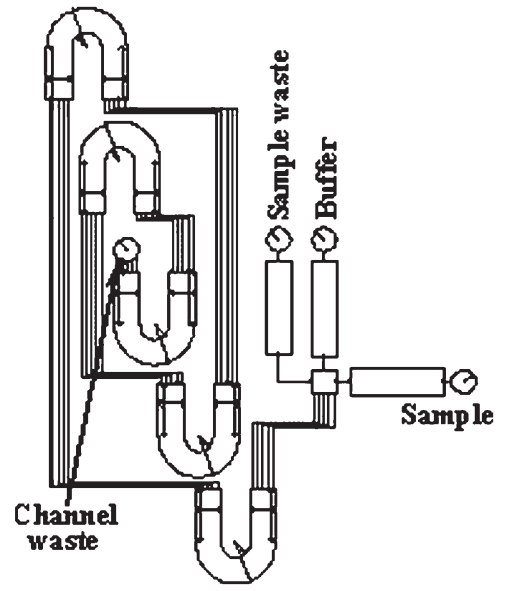

(b)
Fig. 13. (a) Spiral electrophoretic separation microchip [32]. It consists of five turns with continuously decreased radius $(1.9,1.8,1.7,1.6$, and $0.8 \mathrm{~cm})$. Within them, species dichlorofluoroscein flows in the same direction (clockwise). (b) Its hierarchical schematic representation.

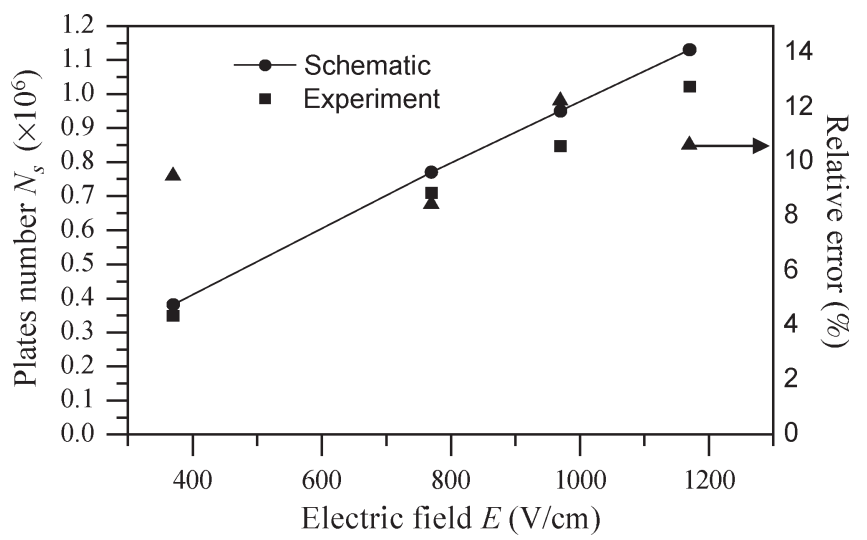

Fig. 14. Comparison of schematic simulation results with the experimental data on theoretical plate number $N_{\mathrm{s}}$ versus electric field $E$. Right axis shows the relative error between simulation and experiments. The linear growth of the plate number $N_{\mathrm{s}}$ with electric field $E$ implies that molecular diffusion is the major dispersion source in such a system.

In Fig. 13, the dispersion of dichlorofluoroscein in a complex spiral separation microchip of five turns is simulated and compared with experimental results [32]. Spiral channels differ from the serpentine in that the skew and variance always increase with the turn number, as the band skew in all turns has the same sense and does not cancel. A scalar index of plate number $N_{\mathrm{S}}$ to characterize the resolving power of the electrophoresis chip is defined $N_{\mathrm{s}}=L_{\text {tot }}^{2} / \sigma^{2}$, where $L_{\text {tot }}$ is the total separation length from injector to the detector. The higher the plate number, the better the separation performance achieved by the chip. The linear growth of the plate number with electric field implies that molecular diffusion is the major dispersion source in such a system (Fig. 14), as molecular diffusion decreases as electric field increases (if joule heating is negligible [28]). The worst case relative error of $12 \%$ is considered acceptably small considering the uncertainties in the knowledge of species diffusivity [32].

Fig. 15 illustrates a hybrid electrophoretic separation microchip [33] and its schematic representation including both spiral and serpentine channels. Due to the difficulty of accounting 


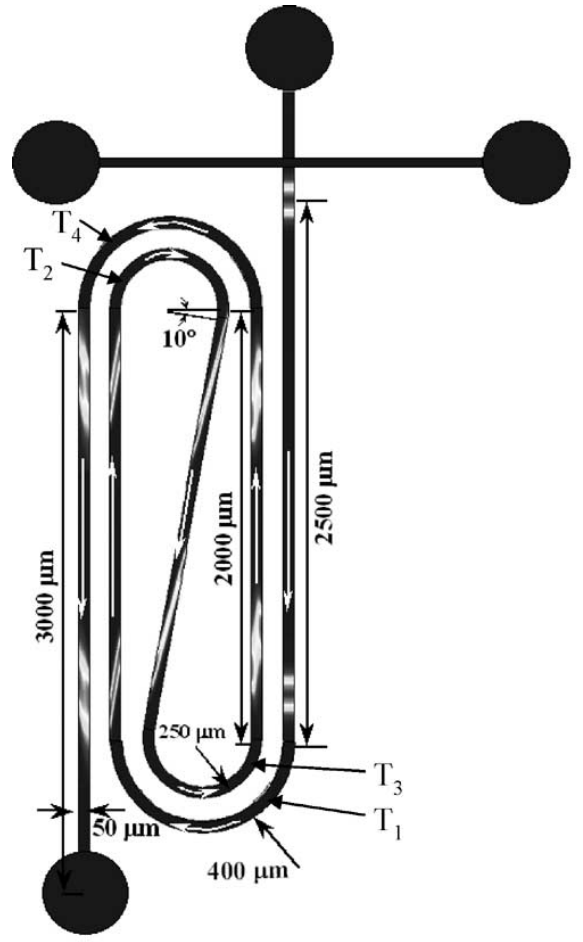

(a)

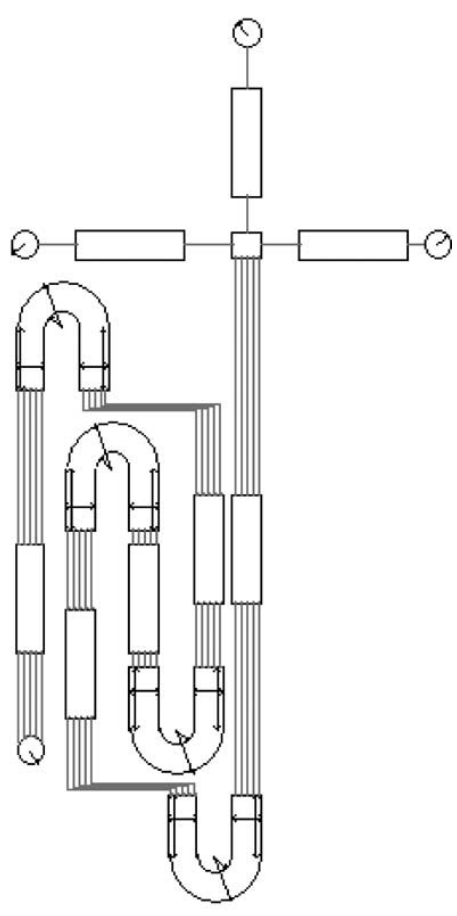

(b)

Fig. 15. (a) Hybrid electrophoretic separation microchip. It consists of both spiral and serpentine channels. Species flows in the clockwise direction in both turns $\mathrm{T}_{1}$ and $\mathrm{T}_{2}$ (spiral topology), thereby $\mathrm{T}_{2}$ strengthens the sharp skew generated by $\mathrm{T}_{1}$. The skew almost persists through the interturn straight channel between $\mathrm{T}_{2}$ and $\mathrm{T}_{3}$ and is significantly canceled out by $\mathrm{T}_{3}$ (serpentine topology). (b) Its hierarchical schematic representation.

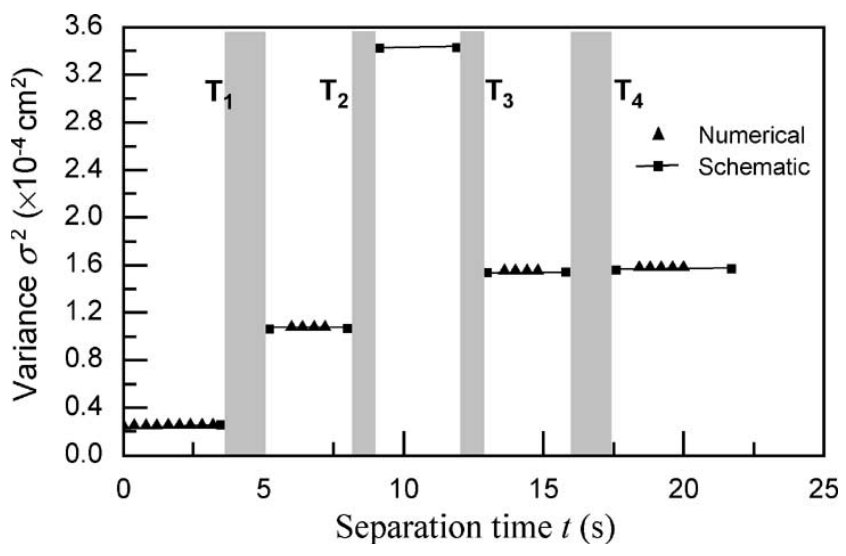

Fig. 16. Comparison of numerical data with dc schematic simulation on variance versus separation time in the hybrid electrophoretic separation microchip. Very sharp skew (see Fig. 15) is generated, and the variance accumulates after turns $T_{1}$ and $T_{2}$ due to their spiral topology. The skew almost persists through the interturn straight channel between $\mathrm{T}_{2}$ and $\mathrm{T}_{3}$ and is significantly canceled out by $\mathrm{T}_{3}$ attributed to their serpentine topology, which, as a result, yields a drastic variance drop after $\mathrm{T}_{3}$.

for the coexisting skew canceling and strengthening effects in such a topology, it has not been effectively investigated since it was proposed [33]. Fig. 16 shows schematic simulation result on the variance of a species band versus time in such a chip, as well as its comparison with numerical data. A low species diffusivity of $D=1 \times 10^{-11} \mathrm{~m}^{2} / \mathrm{s}$ is chosen to analyze the highly convective dispersion that has not been considered by the previous example in Fig. 11 (other properties and parameters are the same as those of sample $a$ in Fig. 11). Highly convective dispersion is practically important for microchip electrophore- sis of the species with low diffusivity, such as the separation of DNA in a gel or sieving matrix [6], [34]. It is shown in Fig. 15 that since species flows in the clockwise direction in both turns $\mathrm{T}_{1}$ and $\mathrm{T}_{2}$ (spiral topology), $\mathrm{T}_{2}$ strengthens the sharp skew generated by $\mathrm{T}_{1}$, leading to a more skewed band and a higher variance. Due to the small species diffusivity, the skew almost persists through the interturn straight channel between $T_{2}$ and $T_{3}$ and is significantly canceled out by $T_{3}$, which, as a result, yields a drastic variance drop in $\mathrm{T}_{3}$ (serpentine topology). However, the skewed band after $\mathrm{T}_{3}$ is overly corrected by $\mathrm{T}_{4}$ and a counterskew is shown afterward. Excellent agreement between the schematic and numerical simulation results with $1 \%$ relative error and tremendous speedup up to $400000 \times$ have been achieved in Fig. 16. This is the first time that the highly convective dispersion in the hybrid electrophoresis microchip at this complexity level has been accurately and efficiently simulated by analytical models.

\section{Future Work}

At present, the separation and mixing models can only be used independently of each other as the mixing occurs in continuous flow of samples, and the separation exploits the transient behavior. To combine them for practical integrated $\mathrm{LoC}$ simulation requires the use of an injector [35]. Additionally, to achieve the canonical assay described in Fig. 2, a reactor model is needed. A simple reactor model has been assembled with the separation and mixing models described above and injector models from [35] to simulate an integrated immunoassay microchip [25] showing the path to our envisioned LoC design methodology. 


\section{CONCLUSION}

Modeling and simulation of EK biofluidic LoC systems (especially complex EK passive micromixers and electrophoretic separation systems) based on the top-down design methodology have been presented. Complex biofluidic LoCs have been geometrically and functionally decomposed into commonly used elements of simple geometry and specific function. Electric and biofluidic pins are proposed to support the communication between adjacent elements. Parameterized models that can accurately capture the element behavior have been implemented in an analog hardware description language (Verilog-A). Thus, a system-level schematic model can be developed for LoC designs for iterative simulations to evaluate the impact of chip topology, element sizes, and material properties on system performance. The simulation employs the Kirchhoff's law and directional signal flow to solve electric and microfluidic networks.

The schematic simulation results of EK passive micromixers and electrophoretic separation microchips have been verified by numerical and experimental data. It has been shown that the proposed behavioral models are able to accurately describe the overall effects of chip topology, material properties, and operational parameters on mixing and separation performance, as well as interactions among elements. Tremendous speedups (up to $20000 \times$ for mixer and $400000 \times$ for electrophoretic separation system) over full numerical simulations have been achieved by schematic simulations using behavioral models, while still maintaining high accuracy (relative error generally less than 5\%). Therefore, our modeling and simulation efforts represent a significant contribution to addressing the need for efficient and accurate modeling and simulation tools to enable optimal designs of integrated biofluidic LoCs.

\section{APPENDIX}

The species band concentration $c(y, z, t)$ within a separation channel is governed by the convection-diffusion equation [10]

$$
\frac{\partial c}{\partial t}+u \frac{\partial c}{\partial z}=D\left(\frac{\partial^{2} c}{\partial z^{2}}+\frac{\partial^{2} c}{\partial y^{2}}\right)
$$

where $y$ and $z$ are the widthwise and axial coordinates, respectively, and $t$ is the separation time from the channel entrance. The width of the species band can be characterized by variance, the square of the standard deviation of the cross-sectional average concentration profile $c_{m}$, which is defined as

$$
\sigma^{2}=\frac{\int_{-\infty}^{\infty}(z-\bar{z})^{2} c_{m} d z}{\int_{-\infty}^{\infty} c_{m} d z}
$$

where $\bar{z}$ is the axial position of the species band's centroid in the channel.

Equation (A1) can be reformulated into a more tractable reduced-dimension form in terms of spatial moments of the species concentration. Such moments are capable of describing the species band's main characteristics such as mass distribution, skew, and variance without solving for detailed concentration distributions. We introduce a new coordinate frame, which moves at the species band's average velocity $U$, and normalize the equation to reduce all variables into dimensionless forms. Define a dimensionless axial coordinate $\xi$, widthwise coordinate $\eta$, and time $\tau$ by

$$
\xi=\frac{(z-U t)}{w} \quad \eta=\frac{y}{w} \quad \tau=\frac{D t}{w^{2}} .
$$

In terms of these dimensionless variables, (A1) is rephrased to the following form in the concentration $c(\eta, \xi, \tau)$ :

$$
\frac{\partial c}{\partial \tau}=\frac{\partial^{2} c}{\partial \xi^{2}}+\frac{\partial^{2} c}{\partial \eta^{2}}-\operatorname{Pe} \chi \frac{\partial c}{\partial \xi}
$$

where $\mathrm{Pe}=U w / D$ is the Peclet number representing the ratio of convection and diffusive transport rates, and $\chi$ is the normalized species velocity relative to the average, given as

$$
\chi(\eta)=\frac{(u-U)}{U} .
$$

We now recast (A4) in terms of spatial moments of the species concentration. If the species band is entirely contained in the channel, (A4) holds effectively over the axial domain $-\infty<\xi<\infty$ (the widthwise domain is $0<\eta<1$ ), such that $c \rightarrow 0$ as $\xi \rightarrow \pm \infty$. Therefore, we can define spatial moments of the species concentration by

$$
c_{p}(\eta, \tau)=\int_{-\infty}^{\infty} \xi^{p} c(\eta, \xi, \tau) d \xi \quad m_{p}(\tau)=\int_{0}^{1} c_{p} d \eta .
$$

Here, $c_{p}$ is the $p$ th moment of the species concentration in the axial filament at $\eta$, and $m_{p}$ is the $p$ th moment of the average concentration of the band. Note that as a consequence of the coordinate transformation (A3), all moments are taken with respect to the moving frame $(\xi, \eta)$. For purposes of simulating analyte dispersion, it suffices to obtain the moments up to the second order. Specifically, $c_{0}$ provides the transverse distribution of the species mass in each axial filament within the channel and $m_{0}$ is the total species mass and can be chosen as $m_{0}=1$ without losing generality. Next, $c_{1}$ gives the axial locations of the centroid of the axial filaments in the species band and, hence, indicates the skew of the band. Then, $m_{1}$, the widthwise average of $c_{1}$, is the axial location of the centroid of the entire species band in the frame $(\xi, \eta)$ and is always zero for this study [10]. Finally, $m_{2}$ can be used to determine the variance $\sigma^{2}$ of the species band by $\sigma^{2}=w^{2}\left(m_{2} / m_{0}-m_{1}^{2} / m_{0}^{2}\right)$.

\section{REFERENCES}

[1] D. R. Reyes, D. Lossifidis, P. A. Auroux, and A. Manz, "Micro total analysis systems-1: Introduction, theory, and technology," Anal. Chem., vol. 74, no. 12, pp. 2623-2636, Jun. 2002.

[2] P. A. Aurouz, D. Lossifidis, D. R. Reyes, and A. Manz, "Micro total analysis systems-2: Analytical standard operations and applications," Anal. Chem., vol. 74, no. 12, pp. 2637-2652, Jun. 2002.

[3] S. C. Jacobson, T. E. McKnight, and J. M. Ramsey, "Microfluidic devices for electrokinetically driven parallel and serial mixing," Anal. Chem., vol. 71, no. 20, pp. 4455-4459, Oct. 1999.

[4] C. A. Emrich, H. J. Tian, I. L. Medintz, and R. A. Mathies, "Microfabricated 384-lane capillary array electrophoresis bioanalyzer for ultrahighthroughput genetic analysis," Anal. Chem., vol. 74, no. 19, pp. 5076-5083, Oct. 2002. 
[5] D. J. Harrison, C. Skinner, S. B. Cheng, G. Ocvirk, S. Attiya, N. Bings, C. Wang, J. Li, P. Thibault, and W. Lee, "From micro-motors to microfluidics: The blossoming of micromachining technologies in chemistry, biochemistry and biology," in Proc. 10th Int. Conf. Solid-State Sensors and Actuators, Sendai, Japan, Jun. 7-10, 1999, pp. 12-15.

[6] C. T. Culbertson, S. C. Jacobson, and J. M. Ramsey, "Dispersion sources for compact geometries on microchips," Anal. Chem., vol. 70, no. 18, pp. 3781-3789, Sep. 1998.

[7] J. B. Knight, A. Vishwanath, J. P. Brody, and R. H. Austin, "Hydrodynamic focusing on a silicon chip: Mixing nanoliters in microseconds," Phys. Rev. Lett., vol. 80, no. 17, pp. 3863-3866, Apr. 1998.

[8] S. Krishnamoorthy and M. G. Giridharan, "Analysis of sample injection and band-broadening in capillary electrophoresis microchips," in Tech. Proc. Int. Conf. Modeling and Simulation Microsystems, San Diego, CA, Mar. 27-29, 2000, pp. 528-531

[9] P. M. S. John, T. Woudenberg, C. Connell, M. Deshpande, J. R. Gilbert, M. Garguilo, P. Paul, J. Molho, A. E. Herr, T. W. Kenny, and M. G. Mungal, "Metrology and simulation of chemical transport in microchannels," in Proc. 8th IEEE Solid-State Sensor and Actuator Workshop, Hilton Head, SC, Jun. 7-11, 1998, pp. 106-111.

[10] Y. Wang, Q. Lin, and T. Mukherjee, "System-oriented dispersion models of general-shaped electrophoresis microchannels," Lab Chip, vol. 4, no. 5, pp. $453-463$, Oct. 2004

[11] J. C. Harley, R. F. Day, J. R. Gilbert, M. Deshpande, J. M. Ramsey, and S. C. Jacobson, "System design of two dimensional microchip separation devices," in Tech. Proc. 5th Int. Conf. Micro Total Analysis Systems (MicroTAS), Monterey, CA, Oct. 21-25, 2001, pp. 63-65.

[12] M. Turowski, Z. J. Chen, and A. Przekwas, "Automated generation of compact models for fluidic microsystems," Analog Integr. Circuits Signal Process., vol. 29, no. 1-2, pp. 27-36, Oct. 2001.

[13] R. Qiao and N. R. Aluru, "A compact model for electroosmotic flows in microfluidic devices," J. Micromech. Microeng., vol. 12, no. 5, pp. 625635, Sep. 2002.

[14] X. C. Xuan and D. Q. Li, "Analysis of electrokinetic flow in microfluidic networks," J. Micromech. Microeng., vol. 14, no. 2, pp. 290-298, Feb. 2004

[15] [Online]. Available: http://www.coventor.com/coventorware/architect/ behavioral models.html

[16] T. H. Zhang, K. Chakrabarty, and R. B. Fair, "Behavioral modeling and performance evaluation of microelectrofluidics-based PCR systems using SystemC," IEEE Trans. Comput.-Aided Des. Integr. Circuits Syst., vol. 23, no. 6, pp. 843-858, Jun. 2004

[17] A. N. Chatterjee and N. R. Aluru, "Combined circuit/device modeling and simulation of integrated microfluidic systems," J. Microelectromech. Syst., vol. 14, no. 1, pp. 81-95, Feb. 2005.

[18] H. Chang, A Top-Down Constraint-Driven Design Methodology for Analog Integrated Circuits. Boston, MA: Kluwer, 1997.

[19] T. Mukherjee, G. K. Fedder, and R. D. S. Blanton, "Hierarchical design and test of integrated microsystems," IEEE Des. Test. Comput., vol. 16, no. 4, pp. $18-27$, Oct.-Dec. 1999

[20] A. J. Pfeiffer, T. Mukherjee, and S. Hauan, "Design and optimization of compact microscale electrophoretic separation systems," Ind. Eng. Chem. Res., vol. 43, no. 14, pp. 3539-3553, Jul. 2004.

[21] — , "Synthesis of multiplexed biofluidic microchips," IEEE Trans. Comput.-Aided Des. Integr. Circuits Syst., 2005. submitted for publication.

[22] R. F. Probstein, Physicochemical Hydrodynamics: An Introduction, 2nd ed. New York: Wiley, 2003.

[23] S. J. Haswell, "Development and operating characteristics of micro flow injection analysis systems based on electroosmotic flow-A review," Analyst, vol. 122, no. 1, pp. R1-R10, Jan. 1997.

[24] S. C. Jacobson, R. Hergenroder, L. B. Koutny, R. J. Warmack, and J. M. Ramsey, "Effects of injection schemes and column geometry on the performance of microchip electrophoresis devices," Anal. Chem., vol. 66, no. 7, pp. 1107-1113, Apr. 1994.

[25] Y. Wang, R. M. Magargle, Q. Lin, J. F. Hoburg, and T. Mukherjee, "System-oriented modeling and simulation of biofluidic lab-on-a-chip," in Proc. 13th Int. Conf. Solid-State Sensors and Actuators, Seoul, Korea, Jun. 5-9, 2005, pp. 1280-1283.

[26] P. Löb, K. S. Drese, V. Hessel, S. Hardt, C. Hofmann, H. Lowe, R. Schenk, F. Schonfeld, and B. Werner, "Steering of liquid mixing speed in interdigital micro mixers-From very fast to deliberately slow mixing," Chem. Eng. Technol., vol. 27, no. 3, pp. 340-345, Mar. 2004.

[27] Y. Wang, Q. Lin, and T. Mukherjee, "A model for laminar diffusion-based complex electrokinetic passive micromixers," Lab Chip, vol. 5, no. 8, pp. 877-887, Aug. 2005.
[28] — - "A model for Joule heating-induced dispersion in microchip electrophoresis," Lab Chip, vol. 4, no. 6, pp. 625-631, Dec. 2004

[29] [Online]. Available: http://www.cadence.com

[30] S. C. Jacobson and J. M. Ramsey, "Electrokinetic focusing in microfabricated channel structures," Anal. Chem., vol. 69, no. 16, pp. 3212-3217, Aug. 1997.

[31] N. H. Chiem and D. J. Harrison, "Microchip systems for immunoassay: An integrated immunoreactor with electrophoretic separation for serum theophylline determination," Clin. Chem., vol. 44, no. 3, pp. 591-598, Mar. 1998.

[32] C. T. Culbertson, S. C. Jacobson, and J. M. Ramsey, "Microchip devices for high-efficiency separations," Anal. Chem., vol. 72, no. 23, pp. 5814-5819, Dec. 2000.

[33] S. K. Griffiths and R. H. Nilson, "Design and analysis of folded channels for chip-based separations," Anal. Chem., vol. 74, no. 13, pp. 2960-2967, Jul. 2002.

[34] B. M. Paegel, L. D. Hutt, P. C. Simpson, and R. A. Mathies, "Turn geometry for minimizing band broadening in microfabricated capillary electrophoresis channels," Anal. Chem., vol. 72, no. 14, pp. 3030-3037, Jul. 2000.

[35] R. Magargle, J. F. Hoburg, and T. Mukherjee, "Microfluidic injector models based on neural networks," IEEE Trans. Comput.-Aided Des. Integr. Circuits Syst., 2005. submitted for publication.

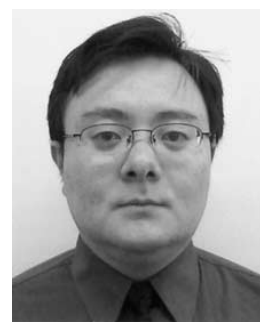

Yi Wang received the B.S. and M.S. degrees in mechanical engineering from Shanghai Jiao Tong University, Shanghai, China, in 1998 and 2001, respectively, and is currently working toward the Ph.D. degree at the Department of Mechanical Engineering, Carnegie Mellon University, Pittsburg, PA.

His research interests include behavioral modeling of microscale biofluidic transport phenomena, system level simulation, and design of integrated labon-a-chip systems.

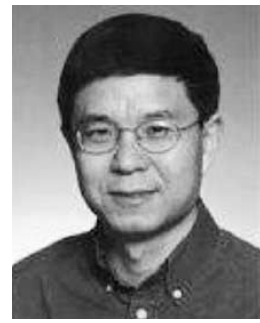

Qiao Lin received the Ph.D. degree in mechanical engineering from the California Institute of Technology, Pasadena, CA, in 1998, with thesis research on kinematics and mechanics of robotic manipulation.

$\mathrm{He}$ conducted postdoctoral research in microelectromechanical systems (MEMS) at the Caltech Micromachining Laboratory from 1998 to 2000 and has since been an Assistant Professor at the Department of Mechanical Engineering, Carnegie Mellon University, Pittsburgh, PA. His research interests are in designing and creating micro/nanosystems, in particular, MEMS and micro/nanofluidic systems, for biomedical applications

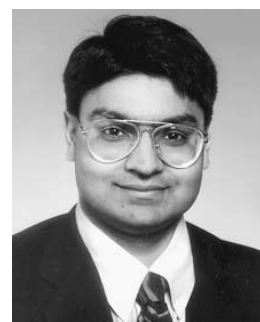

Tamal Mukherjee (S'89-M'95) received the B.S., M.S., and Ph.D. degrees from Carnegie Mellon University, Pittsburgh, PA, in 1987, 1990, and 1995, respectively, all in electrical and computer engineering.

$\mathrm{He}$ is currently a Research Professor at the Department of Electrical and Computer Engineering, Carnegie Mellon University. His research interests include automating the design of analog circuits and microfluidic and microelectromechanical systems. His current work focuses on developing computeraided design methodologies and techniques for integrated microelectromechanical systems, and is involved in modeling, simulation, extraction, and synthesis of mixed domain microsystems. He is also active in the use of these techniques for the design of radio frequency (RF) circuits whose performance is enhanced by micromachining. 